\title{
RETORNOS ANORMALES DE OFERTAS PÚBLICAS INICIALES, EVIDENCIA PARA CHILE: 1993 - 2000
}

\author{
Carlos Maquieira Villanueva* \\ Departamento de Administración \\ Universidad de Chile
}

(Recibido 13 de octubre 2004, aceptado 26 de noviembre 2004)

\section{Resumen}

El objetivo de este trabajo es analizar el comportamiento de las IPO's ocurridas en Chile durante la década de los noventa, por cuanto un gran porcentaje de empresas que se abrieron a Bolsa en ese periodo perdieron gran parte del valor de sus acciones. Para evaluar la rentabilidad anormal que pudo o no haberse generado se examinó el periodo 1993-2000, donde se concentró el mayor número de IPO's en el mercado accionario chileno.

Se utilizaron tres métodos para analizar y corroborar el comportamiento que presentaron las IPO's en el periodo de estudio: Cumulative Adjusted Returns (CAR), Holding Period Returns o Buy and Hold Return (HPR) y el Modelo de Tres Factores de Fama y French. Los resultados obtenidos ante la pérdida de valor que experimentaron casi dos tercios de las acciones de empresas que se abrieron a Bolsa, durante el periodo en estudio, dependen de la metodología utilizada para realizar las diferentes pruebas.

\section{Abstract}

The objective of this study is to analyze the behavior of the IPO's in Chile during the 90's, given the great percentage of Chilean companies that issue their stocks and lost an important amount of their value at that time. Therefore, we will compute the abnormal returns of IPO's for 1993-2000 period.

We consider three methods that have been used to examine and prove the behavior that the IPO's had on the period under study: Cumulative Adjusted Returns (CAR), Holding Period Returns or Buy and Hold Return (HPR), and the Three Factors Model of Fama and French. We find that 67 per cent of the companies lost values for the study period but the results depend on the method used to compute the abnormal behavior of the returns.

\section{Clasificación JEL: G32}

Palabras clave: Oferta pública inicial (IPO), Rentabilidades anormales acumuladas (CAR), Retorno del periodo de tenencia o retorno por tenencia en el largo plazo (HPR), Rentabilidad por comprar y mantener acciones por un periodo determinado, Modelo de tres factores de Fama y French

\footnotetext{
* Escuela de Postgrado, Economía y Negiocios. Facultad de Ciencias Económicas y Administrativas. Universidad de Chile. Diagonal Paraguay 257, of. 1101, Santiago, Chile. Teléfono: $+56(2) 678-3385$. Correo electrónico: cmaquieira@escueladepostgrado.cl
} 
La segunda postura corresponde a la planteada por Fama (1998) y Brav (2000) que mantiene el paradigma de la eficiencia al apuntar que este tipo de anomalías se debe a problemas en la métrica de los rendimientos empleados y a la especificación de los modelos de valoración, los cuales presentan dificultades para valorar adecuadamente las empresas pequeñas y con baja razón "valor libro / valor de mercado". En este contexto, Fama señala que las anomalías relacionadas con rendimientos en el largo plazo son sensibles a la metodología empleada, tendiendo a desaparecer ante razonables cambios en la forma de medirlas.

En esta investigación se estudió la existencia de rendimientos anormales en el primer día de negociación, en una muestra de 36 IPO's, para el periodo comprendido entre julio de 1993 y diciembre de 2000. Los resultados indican que la distribución de los retornos iniciales es muy similar a la que se encuentra en la mayoría de los estudios referidos a este tema, es decir, presentan media positiva, mediana cercana a cero y una relativa asimetría.

En lo que respecta al desempeño a largo plazo que presentaron las IPO's, se demostró lo sensible que es el cálculo de retornos anormales al grupo de control escogido, siendo éstos más severos utilizando al Índice de Precios Selectivo de Acciones (IPSA) como benchmark, en comparación a la utilización del Índice General de Precios y Acciones (IGPA). A lo largo de la investigación se optó por la utilización del IGPA como grupo de control, a diferencia de diversos estudios que han utilizado el IPSA. Lo anterior se debe a que la utilización de un índice selectivo dista mucho de ser un grupo de control idóneo, dado que la mayoría de las IPO's corresponden a empresas pequeñas con una baja razón "valor libro / valor de mercado" y donde sus negocios son inmaduros, pero con altas expectativas de crecimiento.

Se constató la inexistencia de retornos anormales negativos, que resultaron sistemáticos en el periodo en estudio, para una cartera equiponderada de IPOs, mediante la utilización de CAR como también del Modelo de Tres Factores de Fama y French.

En un análisis desagregado, según las características de la emisión de IPO's, las empresas que presentaron un bajo desempeño a largo plazo corresponden a las que emitieron los montos más pequeños durante el primer día. Se constató además, la inexistencia de una relación de causalidad lineal entre el underpricing y el desempeño a largo plazo. De igual forma, las empresas que presentaron un bajo desempeño a largo plazo fueron las pequeñas y con negocios maduros (con una elevada razón "valor libro / valor de mercado"), es decir, con escasas expectativas de crecimiento.

En la sección 2 se revisan las distintas teorías que explican los fenómenos que presentan las IPO's, diferenciando estudios de corto y largo plazo, así como las principales cuestiones metodológicas que afectan la medición de su rentabilidad. La sección 3 describe la base de datos empleada para la muestra y presenta una breve reseña de IPO's realizadas en el mercado Chileno durante la década de los noventa. La sección 4 corresponde a la descripción de la metodología empleada. La sección 5 comenta los resultados obtenidos tanto para el corto como largo plazo mediante el uso de CAR y HPR. La sección 6 comenta los resultados obtenidos para el largo plazo mediante el Modelo de 
Tres Factores de Fama y French. Finalmente, la sección 7 resume los resultados encontrados y presenta las conclusiones.

\section{Estudios previos}

Bajo la existencia de underpricing, las acciones de empresas que se han abierto a Bolsa han sido ofrecidas a los inversionistas a un precio considerablemente inferior al precio al que se han negociado posteriormente en el mercado de valores, midiendo generalmente esta anomalía desde el precio de la oferta pública hasta el precio de cierre del primer día de cotización. Ibbotson (1975) muestra que los retornos iniciales tienen una distribución muy asimétrica, con media positiva y mediana cercana a cero. Otro estudio que revisa y demuestra que los retornos iniciales tienen una distribución asimétrica es el de Ritter (1998), el cual analiza este fenómeno en el mercado norteamericano.

En este mismo sentido, Aggarwal, Leal y Hernández (1993) analizan para Chile esta anomalía. Su muestra correspondió a 19 IPO's efectuadas durante el periodo 1982 - 1990, donde su investigación arrojó un underpricing de $16.3 \%$. Además, se analizó el bajo rendimiento a largo plazo de 28 IPO's efectuadas durante el mismo periodo, considerando una ventana ${ }^{2}$ de 3 años, reportando una rentabilidad anormal de $-23.7 \%$. Ambas conclusiones se encuentran entre los rangos que establece la evidencia internacional reportada por estudios como el de Loughran, Ritter y Rydqvist (1994), quienes encuentran distintos grados de underpricing, y bajo rendimiento a largo plazo, para 38 países.

Por otro lado, donde se han hecho mayores avances es en lo referido al fenómeno del underpricing, donde la asimetría de información es una de sus explicaciones. En efecto, es importante notar que algunas teorías le atribuyen a las empresas un mayor manejo de información con respecto al verdadero valor de la firma que los inversionistas, por lo tanto, se observa que sólo las firmas que son inferiores al promedio van a emitir acciones al precio promedio. Grinblatt y Hwang (1989) sugieren que para distinguirse, las firmas que son mejores al promedio pueden emitir una señal que es costosa $\mathrm{y}$, por lo tanto, no pueden ser imitadas por firmas de menor valor. Esta señal puede ser la venta de sus acciones con un descuento. Lo anterior, según Welch (1989), puede ser recuperado en emisiones posteriores a la IPO. Sin embargo, como argumenta Ritter y Welch (2002), no queda claro por qué el underpricing es una señal más eficiente que cualquier otra.

En otras teorías de asimetría, se destaca que los inversionistas están mejor informados que el emisor respecto a la demanda de acciones. Así, si todos los inversionistas tuvieran la misma información, sólo se observarían emisiones con precios menores al verdadero valor. Sin embargo, en la realidad también se ven emisiones sobrevaluadas y resulta difícil aceptar el supuesto de que todos los inversionistas estén igualmente informados. Para inversionistas que cuentan con distinta información, Rock (1986) propone que existe el underpricing para evitar un Winner's Curse o maldición del ganador, según la cual existen inversionistas informados que participan solamente de algunas ofertas, e inversionistas no informados que participan indiscriminadamente de todas las

2 Número de años durante los cuales se calculan los retornos para las empresas que se han abierto a Bolsa. 
emisiones. El Book Building ${ }^{3}$ es un mecanismo que permite a los inversionistas no informados adjudicarse una subasta en la medida que la acción este sobrevaluada, ésto es llamado Winner's Curse, ya que sólo se gana la subasta cuando la acción está sobrevaluada. De este modo, Rock argumenta que los bancos de inversión tienen interés en mantener a los inversionistas no informados dentro del mercado $y$, por lo tanto, ofrecen un descuento que los compensa por esa desventaja.

Otra teoría, denominada Cascada, supone que los inversionistas observan el comportamiento de otros inversionistas para decidir si comprarán o no, aspecto que si es advertido por los underwriters puede generar una "Cascada Positiva" y asegurar una colocación exitosa si se fija un precio bajo.

En este sentido, un aspecto que se puede destacar de la teoría de asimetría de información, es que el grado de underpricing es función creciente de la asimetría de información.

Entre las teorías de información asimétrica se destaca la postura de Tinic (1988), en la que el underpricing funciona como mecanismo de protección contra futuras acciones legales contra emisores y underwriters. Tinic analiza el underpricing antes y después de la aparición de una norma que aumenta la responsabilidad y enjuiciabilidad de los emisores y encuentra diferencias estadísticamente significativas entre los dos periodos.

La segunda característica irregular de las IPO's son los periodos denominados Hot Issues, en los que se observa una cantidad de IPO's mucho mayor a las que en promedio se efectúan (así como también un underpricing mayor al promedio) y que se deben a un optimismo generalizado en los mercados. Ritter (1984), documentó varios de estos periodos durante la posguerra en Estados Unidos, destacando entre ellos los años 1980-1981 con un 48\%. Posteriormente, junto a Ibbotson y Sindelar (1988), examinó el periodo entre 1960-1987, encontrando un retorno inicial de $16.4 \%$ y corroborando la existencia de este fenómeno.

Finalmente, en el análisis de la tercera anomalía de las IPO's, sobre bajo desempeño en el largo plazo, Ritter (1991) estudia el fenómeno con una ventana de 3 años después de la oferta. Dichas acciones resultaron ser, en promedio, una mala inversión en comparación al resultado que se obtendría al invertir en empresas similares en tamaño y sector. Ibbotson (1975), obtiene resultados similares a los obtenidos por Ritter e intenta explicarlo como un aprovechamiento, por parte de las firmas, de las Cascadas de optimismo en los mercados para financiarse a un bajo costo emitiendo acciones. Esta hipótesis es apoyada por el hecho que el mal desempeño no es generado por el underpricing, sino que se observa especialmente en algunos años y en algunas industrias.

Sin embargo, estudios más recientes han analizado esta última anomalía desde un prisma completamente diferente. En efecto, se ha constatado que existe una variación considerable en las medidas de las rentabilidades anormales y de las pruebas estadísticas, que los investigadores utilizan para detectar el

3 Término que se refiere al libro de registro de potenciales compras y precios dado por quienes desearían participar del remate. Generalmente, de este proceso previo al remate, participan sólo inversionistas institucionales. 
desempeño a largo plazo de las acciones de empresas que se abren a Bolsa. De hecho, Barber y Lyon (1997) y Lyon et al. (1999) han expuesto que el método de cálculo de rendimiento anormal influye tanto en la magnitud de la medida del desempeño, como en el tamaño y poder de las pruebas estadísticas. Fama (1998) apoya esta tesis en sus estudios, y asimismo, la desarrolla formalmente.

Autores como Brav (2000) han puesto de manifiesto que no existe un bajo desempeño a largo plazo de las IPO's. Así, estas empresas obtienen una rentabilidad a largo plazo similar a la obtenida por otras empresas, si la comparación entre éstas se establece según el tamaño ${ }^{4}$ y la razón "valor libro / valor de mercado" de los fondos propios. Éste muestra que los patrones de rentabilidad de las empresas que lievan a cabo IPO's no son diferentes de las que ya participan del mercado bursátil, donde la existencia de retornos anormales reportados en investigaciones previas era motivada por los métodos utilizados para inferir la existencia de un bajo desempeño a largo plazo.

De este modo, existen diversas cuestiones metodológicas que afectan a la estimación de las rentabilidades anormales a largo plazo:

- Respecto al método de Rentabilidades Anormales Acumuladas (CAR) y al de Retornos por Tenencia en el Largo Plazo (HPR), Barber y Lyon (1997), Lyon et al. (1999) y Fama (1998), analizan las alternativas utilizadas para la medición de las rentabilidades anormales, pero no obtienen una conclusión única con respecto al método preferido. Algunos de estos trabajos, como Fama (1998), justifican la utilización del CAR, dado que existe un mayor conocimiento de las propiedades de la distribución de éstos y de las pruebas estadísticas empleadas. Así, Brav (2000) constata que la rentabilidad anormal, como diferencia entre la rentabilidad de una estrategia de comprar y mantener acciones de IPO's durante un periodo determinado y la rentabilidad de una estrategia similar realizada sobre una inversión alternativa, tiende a sobreestimar el bajo desempeño a largo plazo de las IPO's. Sin embargo, Barber y Lyon (1997) destacan la ventaja del HPR para medir la experiencia del inversionista, ya que la utilización de rentabilidades mensuales acumuladas no mide adecuadamente la rentabilidad obtenida al mantener un título por un largo periodo de tiempo. Según estos autores, la rentabilidad obtenida por un inversionista en el largo plazo, es mejor aproximada por la capitalización compuesta de rentabilidades simples a corto plazo, constatando además que rentabilidades anormales acumuladas son un predictor sesgado del HPR. Por consiguiente, dado que no existe unicidad en los planteamientos sobre el tema, en esta investigación se utilizarán tanto los retornos por tenencia en el largo plazo (36 meses), como también los CAR.

- En cuanto a la utilización de ponderadores para las carteras en estudio, Loughran y Ritter (2000) muestran que la elección del esquema de ponderación es un punto relevante. Si el interés se centra en cuantificar el cambio en la riqueza media del inversionista como consecuencia de un determinado evento, el método correcto sería una ponderación por capitalización bursátil. Sin embargo, si el interés se encuentra en las implicaciones de una potencial mala valoración por parte del mercado, un esquema basado en rentabilidades igualmente ponderadas será más apropiado. En la presente investigación se utilizará

4 Se clasifica según el Patrimonio Bursátil, que se calcula como el número de acciones en circulación multiplicado por el precio de cierre. 
un esquema equiponderado para las carteras compuestas por IPO's, tanto en el cálculo del CAR, HPR y como en el Modelo de Tres Factores de Fama y French.

- En cuanto a la elección de un grupo de control o benchmark, Barber y Lyon (1997) constatan que la utilización de carteras compuestas por empresas de similar tamaño y razón "valor libro / valor de mercado" de los fondos propios, producen pruebas estadísticas bien especificadas. Además, las pruebas estadísticas observan sesgos significativos cuando las rentabilidades anormales son estimadas utilizando una cartera de referencia, como por ejemplo un índice de mercado. En este análisis se utilizó el IGPA como grupo de control, sin embargo, en el Modelo de Tres Factores es posible inferir la rentabilidad anormal a partir de la rentabilidad excesiva sobre el IGPA como también sobre carteras basadas en el tamaño y razón "valor libro / valor de mercado".

Tal como señala Fama (1998), todos los métodos utilizados para la estimación de rentabilidades anormales están sujetos a problemas derivados de la mala especificación de los modelos y ningún método es capaz de minimizar esos problemas para toda clase de eventos. Incluso, en modelos como el Tres Factores de Fama y French y los que utilizan diversos grupos de control, se da lugar a diferentes estimaciones de las rentabilidades anormales (Fama 1998). Por este motivo, en esta investigación se ha optado por medir el desempeño de las IPO's por los métodos clásicos (CAR y HPR), y además, incorporar al análisis del Modelo de Tres Factores de Fama y French.

\section{Breve reseña y muestra de las IPO's en la década de los 90's}

En este estudio se ha empleado una base de datos integrada por las empresas que comenzaron a cotizar en la Bolsa de Comercio de Santiago a lo largo del periodo 1993-1998, coincidiendo con el auge de este mercado y concentrando el mayor número de empresas con interés de ingresar a la Bolsa.

Es importante notar que del número total de empresas que comenzaron a cotizar por primera vez en la Bolsa de Comercio de Santiago en los años 90 's, sólo un tercio de ellas pudo sostener o incrementar el precio al que fueron colocadas sus acciones en el primer día de transacción. Los dos tercios restantes perdieron la mitad de su valor desde ese primer día hacia fines de los 90's, incluso algunas de ellas salieron del mercado poco tiempo después. La oleada de aperturas que se sucedieron desde inicios de los 90's respondió a condiciones propias de una economía que crecía a una tasa promedio del $7.0 \%$ anual y a un entorno político estable que generaba incentivos al Mercado de Valores (mediante reglamentaciones favorables a la inversión en él como el beneficio tributario del 57 bis $^{5}$ ).

Esta oleada de aperturas finalizó abruptamente en el año 1998, en medio del inicio de un largo periodo de contracción económica, al cual el Mercado de Valores no era ajeno. En dicho año se inscribieron 30 firmas nuevas en el registro de valores de la $\mathrm{SVS}^{6}$, que representa el paso previo a la colocación inicial. Sin embargo, ninguna de ellas llevó sus papeles a la rueda.

5 El 57 bis corresponde a un beneficio tributario que pernitió a los contribuyentes que tuvieran inversiones en acciones de pago de primera emisión, realizar una rebaja a la base imponible del Impuesto Global Complementario o Impuesto Único de Segunda Categoría.

6 Superintendencia de Valores y Seguros de Chile (SVS). 
Del total de IPO's llevadas a cabo en el periodo elegido, ${ }^{7}$ se seleccionaron 36 de un total de 45 de las contabilizadas inicialmente. El criterio de selección fue su porcentaje promedio mensual de presencia bursátil ${ }^{8}$ durante el periodo, que fue fijado en un $20 \%$.

En el caso de los modelos CAR y HPR para cada acción, se emplean los precios de cierre a lo largo de los 3 primeros años después de efectuada la IPO, además de contar con el volumen ofrecido durante el primer día de colocación, precio de oferta obtenido a partir del prospecto, ${ }^{9}$ como también el precio medio de la cotización y el monto inicial colocado durante el primer día, entre otros datos. Para el análisis del Modelo de Tres Factores de Fama y French se utilizó además la rentabilidad mensual de cada título accionario que cotizó en la Bolsa de Comercio de Santiago para el periodo que comprende este estudio; considerando las emisiones de pago, la reinversión de dividendos y repartos. Cabe destacar, que en estos tres modelos no se consideró la rentabilidad inicial de la emisión al examinar el desempeño a largo plazo de las IPO's.

De las 36 ofertas públicas de la muestra, participaron 9 Bancos underwriters, entendiendo éstos como "agentes coordinadores" $\mathrm{y} / \mathrm{o}$ "agentes colocadores" de la oferta. Cabe señalar, que en un contrato de underwriting, el Banco de Inversión se compromete a colocar una cantidad determinada de acciones y, en caso de no poder venderlas en el mercado secundario se obliga a comprar el excedente.

La lista de estas 36 IPO's utilizadas puede verse en la Tabla 1. De estas ofertas, todas manejan datos de corto plazo, mientras que sólo 35 tienen datos de largo plazo. ${ }^{10}$ Es importante destacar dentro de la muestra, que la oferta que presenta el mayor volumen colocado durante el primer día es Quiñenco, mientras que la que presenta el menor volumen colocado es la empresa Seguravita S.A. Es posible apreciar una gran dispersión en los montos colocados durante el primer día de la IPO entre las distintas empresas.

Durante el periodo estudiado, siempre existe actividad en el mercado de las IPO's, permaneciendo prácticamente constante el número de ingreso de firmas a la Bolsa durante los años 1993-1997, finalizando abruptamente en el año 1998. El número de ingresos a la Bolsa de Comercio de Santiago, según los años a lo largo del periodo analizado puede apreciarse en la Tabla 2. Los años de auge de IPO's corresponderían a los años 1994 a 1997, de éstos, los dos últimos años presentan los mayores montos emitidos.

7 La lista completa de IPO's, así como sus datos tuvieron como fuente la base de datos obtenida de la Bolsa de Comercio de Santiago. Se eliminaron las ofertas de acciones preferentes y las emitidas subsidiariamente por una empresa que anteriormente ya cotizara en la Bolsa de Comercio de Santiago.

8 Número de días en que se registraron transacciones, expresado como porcentaje del número de días hábiles del mes.

9 Así como también el precio mínimo, que aparece en las Noticias Bursátiles avisando el remate voluntario de acciones inscritas de emisión primaria, previo a la fecha de colocación.

10 Hemos debido eliminar de la muestra a largo plazo a la empresa Cruzsalud por no contar con los datos suficientes en la base de datos para el análisis de largo plazo. Por esta razón, en esa etapa del estudio sólo se mencionan 35 de las 36 empresas iniciales. 
Tabla 1. Fecha, volumen durante el primer día y datos de corto-largo plazo de las IPO's durante 1993-2000

\begin{tabular}{|c|c|c|c|c|c|}
\hline $\mathrm{N}^{o}$ & Fecha & Empresa. & $\begin{array}{c}\text { Monto } \\
\text { Emitido(\$) }\end{array}$ & $\begin{array}{l}\text { Datos } \\
\text { de C.P. }\end{array}$ & $\begin{array}{r}\text { Datos } \\
\text { de L. P. }\end{array}$ \\
\hline 1 & $12 / 07 / 93$ & CINTAC & $\$ 105.569 .250$ & $\sqrt{ }$ & $\sqrt{ }$ \\
\hline 2 & $15 / 07 / 93$ & SOPRAVAL & $\$ 1.546 .335 .777$ & $\sqrt{ }$ & $\sqrt{ }$ \\
\hline 3 & $11 / 08 / 93$ & CORESA & $\$ 867.275 .000$ & $\sqrt{ }$ & $\sqrt{ }$ \\
\hline 4 & $3 / 09 / 93$ & BATA & $\$ 2.198 .046 .600$ & $\sqrt{ }$ & $\sqrt{ }$ \\
\hline 5 & $5 / 10 / 93$ & CADENA & $\$ 351.000 .000$ & $\sqrt{ }$ & $\sqrt{ }$ \\
\hline 6 & $13 / 10 / 93$ & STA ISABEL & $\$ 251.923 .800$ & $\sqrt{ }$ & $\sqrt{ }$ \\
\hline 7 & $16 / 11 / 93$ & ZALAQUETT & $\$ 640.045 .000$ & $\sqrt{ }$ & $\sqrt{ }$ \\
\hline 8 & $29 / 04 / 94$ & COCHRANE & $\$ 537.972 .500$ & $\sqrt{ }$ & $\sqrt{ }$ \\
\hline 9 & $9 / 06 / 94$ & REBRISA-A & $\$ 2.448 .000$ & $\sqrt{ }$ & $\sqrt{ }$ \\
\hline 10 & $4 / 08 / 94$ & SEGURAVITA & $\$ 1.300 .000$ & $\sqrt{ }$ & $\sqrt{ }$ \\
\hline 11 & $9 / 11 / 94$ & INVERCAP & $\$ 165.645 .035$ & $\sqrt{ }$ & $\sqrt{ }$ \\
\hline 12 & $9 / 11 / 94$ & TERRANOVA & $\$ 403.688 .920$ & $\sqrt{ }$ & $\sqrt{ }$ \\
\hline 13 & $15 / 11 / 94$ & BICECORP & $\$ 11.749 .352 .402$ & $\sqrt{ }$ & $\sqrt{ }$ \\
\hline 14 & $18 / 11 / 94$ & INFODEMA & $\$ 440.650 .000$ & $\sqrt{ }$ & $\sqrt{ }$ \\
\hline 15 & $19 / 12 / 94$ & CRUZSALUD & $\$ 3.850 .000 .000$ & $\sqrt{ }$ & \\
\hline 16 & $26 / 05 / 95$ & BESALCO & $\$ 128.747 .520$ & $\sqrt{ }$ & $\sqrt{ }$ \\
\hline 17 & $5 / 07 / 95$ & SECURITY & $\$ 3.159 .005 .000$ & $\sqrt{ }$ & $\sqrt{ }$ \\
\hline 18 & $6 / 12 / 95$ & DUNCANFOX & $\$ 1.530 .000$ & $\sqrt{ }$ & $\sqrt{ }$ \\
\hline 19 & $12 / 12 / 95$ & CONOSUR & $\$ 109.009 .500$ & $\sqrt{ }$ & $\sqrt{ }$ \\
\hline 20 & $26 / 12 / 95$ & $\mathrm{CBI}$ & $\$ 3.675 .065 .790$ & $\sqrt{ }$ & $\sqrt{ }$ \\
\hline 21 & $29 / 04 / 96$ & QUINTEC & $\$ 133.760 .530$ & $\checkmark$ & $\sqrt{ }$ \\
\hline 22 & $4 / 09 / 96$ & ENERQUINTA & $\$ 13.888 .430 .000$ & $\sqrt{ }$ & $\sqrt{ }$ \\
\hline 23 & $17 / 10 / 96$ & FROWARD & $\$ 1.390 .235$ & $\sqrt{ }$ & $\sqrt{ }$ \\
\hline 24 & $5 / 11 / 1996$ & SANTANGRUP & $\$ 9.325 .675 .120$ & $\checkmark$ & $\sqrt{ }$ \\
\hline 25 & $21 / 11 / 96$ & PARIS & $\$ 19.999 .999 .950$ & $\sqrt{ }$ & $\sqrt{ }$ \\
\hline 26 & $26 / 11 / 96$ & FALABELLA & $\$ 2.150 .571 .400$ & $\sqrt{ }$ & $\sqrt{ }$ \\
\hline 27 & $12 / 12 / 96$ & DETROIT & $\$ 2.209 .583 .050$ & $\sqrt{ }$ & $\sqrt{ }$ \\
\hline 28 & $30 / 12 / 96$ & D\&S & $\$ 21.150 .000 .000$ & $\sqrt{ }$ & $\sqrt{ }$ \\
\hline 29 & $9 / 05 / 97$ & SM UNIMARC & $\$ 6.167 .000 .000$ & $\sqrt{ }$ & $\sqrt{ }$ \\
\hline 30 & $27 / 05 / 97$ & CB CAPITAL & $\$ 3.721 .376$ & $\sqrt{ }$ & $\sqrt{ }$ \\
\hline 31 & $27 / 05 / 97$ & CB INVERIN & $\$ 6.285 .411$ & $\sqrt{ }$ & $\sqrt{ }$ \\
\hline 32 & $27 / 05 / 1997$ & $\mathrm{P} \& S$ & $\$ 44.543 .805$ & $\sqrt{ }$ & $\sqrt{ }$ \\
\hline 33 & $25 / 06 / 97$ & QUIÑENCO & $\$ 21.706 .311 .060$ & $\sqrt{ }$ & $\sqrt{ }$ \\
\hline 34 & $4 / 12 / 97$ & FASA & $\$ 1.908 .521 .566$ & $\sqrt{ }$ & $\sqrt{ }$ \\
\hline 35 & $18 / 12 / 97$ & EMBONOR-A & $\$ 14.400 .000 .000$ & $\checkmark$ & $\sqrt{ }$ \\
\hline 36 & $14 / 04 / 98$ & QUILICURA & $\$ 11,254.713$ & $\sqrt{ }$ & $\sqrt{ }$ \\
\hline
\end{tabular}


Tabla 2. Año, cantidad, volumen expresado en pesos de diciembre de 2000 y porcentaje

\begin{tabular}{|cccc|}
\hline Año & $\mathrm{N}^{o}$ & Monto Emitido(\$) & $\%$ \\
\hline 1993 & 7 & $\$ 8.456 .709 .781,0$ & $5,36 \%$ \\
1994 & 8 & $\$ 21.331 .533 .619,0$ & $13,51 \%$ \\
1995 & 5 & $\$ 8.230 .811 .316,1$ & $5,21 \%$ \\
1996 & 8 & $\$ 74.247 .264 .767,5$ & $47,02 \%$ \\
1997 & 7 & $\$ 45.614 .385 .379,2$ & $28,89 \%$ \\
1998 & 1 & $\$ 11.254 .713,0$ & $0,01 \%$ \\
\hline Total & 36 & $\$ 157.891 .959 .576$ & $100,00 \%$ \\
\hline
\end{tabular}

El sector que llevó a cabo mayor cantidad de IPO's corresponde al de Inversiones e Inmobiliarias, con un total de 16 ofertas realizadas; seguido muy por debajo por el sector Comercial y Distribuidores, con 7 IPO's, que sumadas a las anteriores representan más del $50 \%$ del total, fenómeno que usualmente se presenta en economías que tienen el nivel de tasas de crecimiento experimentado por Chile durante el periodo en estudio (7\%).

Siguiendo la metodología de Ritter (1991), se intentó construir una muestra con empresas de igual tamaño, sector u otros parámetros considerados relevantes, intentando utilizar empresas que ya cotizaban en la Bolsa de Comercio de Santiago como grupos de control en inferencias sobre la existencia de retornos anormales. Lamentablemente, esto no fue posible debido al escaso número de empresas que cotizan sus acciones en la Bolsa, el cual fue insuficiente para construir distintas carteras representativas. A falta de esta herramienta, se utilizo como benchmark el IGPA.11

\section{Metodología}

Para evaluar el desempeño de una oferta pública en el corto plazo se mide el retorno obtenido en el primer día de la colocación como la tasa de variación entre el precio de la oferta pública (precio contenido en el prospecto o en el aviso de remate de instrumentos inscritos de emisión primaria) y el precio medio del primer día de la apertura bursátil, ${ }^{12}$ para las empresas que componen la muestra.

En el cálculo de los retornos iniciales se utilizó el precio de colocación que aparece publicado en el prospecto de emisión. Debido a que en algunos casos estos aparecen de manera muy anticipada a la fecha de colocación, se le dió prioridad al precio mínimo que aparece unos días antes de la colocación en el aviso de remate de instrumentos inscritos de emisión primaria, en las Noticias Bursátiles de la Bolsa de Comercio de Santiago. ${ }^{13}$ Además, se utilizó el precio

11 Además, se utilizó el IPSA para evidenciar lo sensible que resulta el cálculo de retornos anormales a los diversos índices de mercado utilizados como benchmark, más aún en el caso del IPSA en Chile, dado el sesgo que le genera el sector eléctrico.

12 El cual aparece en el Informativo Bursátil Diario, el día que se efectuó la oferta inicial, en el resumen de transacciones de colocaciones primarias de acciones.

13 Recolectados de los Informativos Bursátiles Diarios, publicados por la Bolsa de Comercio de Santiago. 
medio del remate que aparece el día de la IPO en el mismo informativo.

Para evaluar el desempeño de una oferta pública en el largo plazo, las rentabilidades de las acciones se calculan dependiendo del modelo utilizado. En este trabajo se utilizaron tres formas distintas de evaluar los rendimientos anormales:

- El primero corresponde a la medición de los Retornos Ajustados Acumulados, mediante el uso de una cartera temporal (Calendar-Time ${ }^{14}$ ). Para éste, se utilizó un periodo de hasta 36 meses desde la apertura a Bolsa de cada IPO.

- El segundo corresponde al cálculo de la rentabilidad anormal a largo plazo, como diferencia entre la rentabilidad de una estrategia consistente en la compra de acciones al final del primer día de negociación y mantenerlas durante un periodo de 36 meses y la rentabilidad de una estrategia similar realizada sobre la posibilidad de invertir en un índice de mercado (IGPA). Para ello, se utilizó el Retorno por Tenencia en el Largo Plazo (HPR), además de un índice de riqueza que permitiese captar y evaluar el desempeño, tanto de las acciones individuales, como de las carteras conformadas según diversos criterios.

- El tercero corresponde a la utilización del Modelo de Tres Factores de Fama y French $(1992,1993)$. Para el análisis se utilizó un periodo comprendido entre julio de 1993 y diciembre de 2000. A diferencia de los dos métodos anteriores, se calculó la rentabilidad mensual de una cartera de IPO's que presentaban acciones efectivamente transadas durante cada mes del periodo señalado.

\subsection{Retornos ajustados acumulados (CAR) mediante el uso de una una cartera temporal}

Esta forma de evaluar el desempeño de largo plazo consiste en calcular el CAR para una cartera equiponderadamente invertida en todas las IPO's. El Retorno Mensual Ajustado de una acción $i$ se calcula como el retorno mensual de esa acción menos el retorno mensual de índice de mercado (IGPA), durante intervalos de 21 dias, ${ }^{15}$ correspondiente a un mes $(t=1)$. Todos los retornos utilizados en este estudio son Retornos Reales. ${ }^{16}$

$$
R_{i, t}^{*}=R_{i, t}-R_{I G P A, t} .
$$

Para una cartera temporal de $n$ acciones, el Retorno Mensual Ajustado Promedio durante un mes será el promedio aritmético de los retornos ajustados de ese mes para todas las acciones:

$$
\mathrm{AR}_{t}=\frac{1}{n} \sum_{i=1}^{36} R_{i, t}^{*}
$$

14 Procedimiento que permite conformar una cartera de empresas que han llevado a cabo una IPO dentro de una ventana establecida, permitiendo acomodar las acciones que dejan de cotizar a medida que transcurre el periodo en estudio.

15 Se definió un mes como 21 días consecutivos de cotización, de manera que el primer mes incluye los días 1-21 de la serie de precios de cierre, el segundo mes corresponde a los días $22-42$, etc.

16 Los precios de cierre diarios fueron divididos previamente por la UF correspondiente a esa fecha, para obtener los retornos reales. 
Esto implica que debe acomodarse mensualmente la cartera para compensar las acciones que dejan de cotizar antes del mes 36. Finalmente, el CAR entre los meses $s$ y $q$ será:

$$
\mathrm{CAR}_{s}^{q}=\sum_{s}^{q} \mathrm{AR}_{t} .
$$

En este trabajo no se ajustan los retornos por los "betas" $(\beta)$ de cada acción. Cabe señalar que, al ajustar los retornos por un índice de mercado sin contemplar las diferencias en los "betas" se produce un sesgo de ajuste. Si el índice de mercado tiene un retorno mayor a la tasa libre de riesgo, las empresas con alto riesgo de mercado, es decir, altos "betas", van a tener altos rendimientos aún después de ajustarlos por el índice, pero estos altos rendimientos no necesariamente indican que tuvo un rendimiento extraordinariamente bueno, sino que tiene una compensación por su riesgo sistemático. Al ajustar esta acción por el índice, sin contemplar el " $\beta$ ", se estará sobreestimando su desempeño. Si por el contrario, el mercado rinde menos que la tasa libre de riesgo, se estará subestimando el desempeño de una acción con un " $\beta$ " mayor a uno. De la misma manera, cuando el " $\beta$ " es menor que uno, los retornos estarán subestimados si el market premium es positivo y sobrestimado cuando el market premium sea negativo. ${ }^{17}$ Ritter (1991) no tiene este problema ya que utiliza empresas comparables en lugar de un índice de mercado. Al considerar la posibilidad de ajustar incluyendo los betas, esto se haría de la siguiente forma:

$$
R_{i, t}^{*}=R_{i, t}-R_{f}-\beta\left(\mathrm{E}\left[R_{\text {market }}\right]-R_{f}\right) .
$$

El problema radica en que el cálculo de los "betas" sólo puede realizarse sobre la misma muestra, con lo cual el retorno ajustado descrito por esta fórmula es simplemente el error de una regresión y, por lo tanto, está forzado a tener esperanza cero a lo largo de todo el periodo.

Adicionalmente, el cálculo recursivo de los "betas" en los primeros años de vida después de una IPO es muy inestable. Ibbotson (1975) constata que los "betas" son en promedio mayores a uno y decrecen con el tiempo a partir de la fecha de emisión.

\subsection{Retornos por tenencia en el largo plazo (HPR)}

El Holding Period Return (HPR) es el resultado de comprar una acción al cierre del primer día y venderla a los tres años o al momento en que se retira de cotización. ${ }^{18}$ Los HPR durante los primeros 3 años de la acción se definen

17 Bajo el CAPM se cumple que $E\left(R_{i}\right)=\beta_{i} \times r_{m}$ (definido $r_{m}=R_{m}-R_{f}$ y $r_{i}=$ $\left.R_{i}-R_{f}\right)$. Ell retorno en exceso de lo que predice la teoría es $E\left(R_{i}\right)=\beta_{i} \times r_{m}$, mientras que ajustando únicamente por el índice de mercado queda un exceso igual a $r_{i}-r_{m}$. La diferencia entre el exceso de retorno estimado y el verdadero es: Actual - real $=\left(\beta_{i}-1\right) r_{m}$. Aquí está claro que para betas mayores a uno y market premium positivo se está sobreestimando el rendimiento de la acción en cuestión.

18 El Holding Period Return a los tres años o al momento en que se retira de cotización, a lo largo del estudio son calculados como $H P R_{i}=\Pi_{t=1}^{36}\left(1+R_{i, t}\right)-1$. De esta forma se determinó el retorno bruto obtenido. 
como:

$$
H P R_{i}=\prod_{t=1}^{36}\left(1+R_{i, t}\right)
$$

Para ajustar e interpretar estos retornos se define la riqueza relativa $(W R)$ de la acción $i$ como:

$$
W R_{i}=\frac{\left(1+H P R_{\dot{i}}\right)}{\left(1+H P R_{I G P A, i}\right)}
$$

Donde $H P R_{I G P A, i}$ es el Holding Period Return del indice IGPA durante los primeros 36 meses de vida de la acción $i$.

La interpretación de los $W R$ es la siguiente, un $W R>1$ indica que esa acción o cartera obtuvo buen desempeño en el largo plazo, es decir, obtuvo retornos mayores al benchmark.

Adicionalmente, se realizó un análisis de series de corte transversal y de tiempo, para analizar el desempeño de largo plazo y así encontrar las relaciones entre retornos en el largo plazo y características de emisión (tamaño, retornos iniciales, sector económico, año de emisión y underwriter). Así como una regresión para observar la significancia del retorno inicial, como del desempeño del benchmark sobre el desempeño a largo plazo de las IPO's.

\subsection{Modelo de Tres Factores de Fama y French (1992, 1993)}

El Modelo de Tres Factores de Fama y French (1993) ha sido utilizado por diversos trabajos como los realizados por Womack (1996), Loughran y Ritter (1995) y Brav (2000), para estimar la rentabilidad anormal durante un determinado horizonte de inversión. Asumiendo que este periodo es el comprendido entre julio de 1993 y diciembre de 2000 , se ha calculado para cada mes la rentabilidad de una cartera compuesta por todas aquellas empresas de la muestra escogida. Esta rentabilidad mensual es utilizada para estimar la siguiente regresión:

$$
R_{p, t}-R_{f, t}=\alpha_{p}+\beta_{p}\left(R_{m, t}-R_{f, t}\right)+s_{p} * S M B_{t}+h_{p} * H M L_{t}+\varepsilon_{p, t} .
$$

Donde $R_{p, t}$ es la Rentabilidad Mensual simple de una cartera compuesta por todas aquellas empresas que han realizado una IPO's en el periodo de estudio; $R_{f, t}$ es la Tasa Libre de Riesgo; $R_{m, t}$ es la Rentabilidad Mensual del IGPA; $S M B_{t}$ es la diferencia en la rentabilidad entre dos carteras compuestas por empresas de tamaño pequeño (cartera de empresas que tienen un valor de mercado de los fondos propios inferior al valor promedio de todas las empresas admitidas a cotización en la Bolsa de Comercio de Santiago) y de gran tamaño (cartera de empresas que tienen un valor de mercado de los fondos propios superior al valor promedio de todas las empresas admitidas a cotización en la Bolsa de Comercio de Santiago); $H M L_{t}$ es la diferencia en la rentabilidad de dos carteras compuestas por empresas con una elevada razón "valor libro / valor de mercado" de los fondos propios (cartera compuesta por todas las empresas cuya razón está dentro del $30 \%$ superior de todas las empresas cotizadas) frente a empresas con un bajo valor para esta misma razón (cartera formada por las empresas con una razón que se encuentra dentro del $30 \%$ inferior del total de 
empresas considerado). ${ }^{19}$ La significancia estadística del término independiente " $\alpha_{p}$ ", proporciona una prueba de la hipótesis nula que la rentabilidad anormal mensual media es cero.

A diferencia de otros modelos similares, como lo son el CAPM de Merton y el ATP de Ross, el Modelo de Tres Factores de Fama y French incorpora en su análisis variables que van en directa relación con la cartera en estudio (tamaño y razón "valor libro / valor de mercado" (BE/ME)).

\section{Desarrollo en el corto plazo}

Los retornos iniciales de las 36 IPO's para las cuales hay datos son muy bajos en comparación a los encontrados en otros países. Tal como en los países estudiados por Loughran, Ritter y Rydqvist (2001) en los que se reporta un underpricing mayor a $5.4 \%$, nuestro país no presenta una excepción, ya que para el periodo de estudio se encontró un underpricing en promedio igual a $6.77 \%$. El titulo accionario de menor retorno inicial resultó ser la acción CB Capital, que cayó un $26.48 \%$, mientras que la acción de Conosur tuvo el máximo retorno inicial, que correspondió a $45.35 \%$.

El bajo promedio de underpricing resulta sorprendente, especialmente en el contexto de las teorías de información asimétrica, ya que a priori no hay razón para creer que la asimetría de información será menor en Chile que en otros países donde también se realizaron estos estudios. La Figura 1 muestra la distribución de los retornos iniciales de las 36 empresas para las cuales existen datos de corto plazo.

Figura 1. Distribución del underpricing para las 36 observaciones
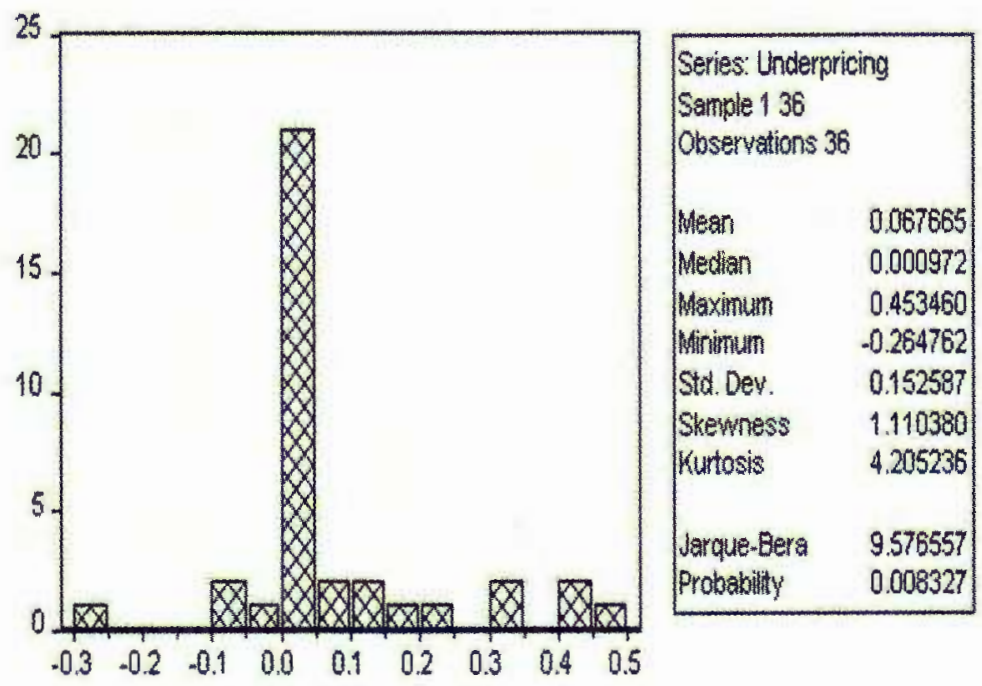

En general, la distribución de los retornos iniciales es parecida a la encontrada en la mayoría de los estudios del tema, es decir, tiene media positiva, mediana

19 Fama, E. y K. French (1993), página 8. 
cercana a cero y una asimetría relativa. Dicha asimetría puede explicarse porque la distribución de retornos iniciales está truncada en el "-1" (no se puede perder más del $100 \%$ del precio de una acción), mientras que la ganancia puede ser mucho mayor al 100\%. En estudios que incluyen más empresas (Ritter (1991)) se aprecian los resultados más extremos en el rango $-99 \%$ a $3964 \% .20$

Como se observa en la Tabla 3, parece haber' una diferencia importante en el grado de underpricing a través de distintos sectores económicos. Encabeza la lista el sector Agropecuario y Forestal con un retorno del 33.85\% para el primer día, existiendo altos retornos también en el sector de Productos Diversos $(24.85 \%)$. En otros sectores los retornos iniciales son muy bajos, siendo cercanos a cero en el caso de Textiles y Vestuario (0.01\%) y Servicios $(0.00 \%){ }^{21}$

Tabla 3. Sector, cantidad y underpricing

\begin{tabular}{|ccc|}
\hline Sector & $\mathrm{N}^{\circ}$ & Underpricing(\%) \\
\hline Agropecuarias y Forestales & 1 & $33,85 \%$ \\
Alimenticias y Bebidas & 2 & $1,15 \%$ \\
Comerciales y Distribuidoras & 7 & $6,86 \%$ \\
Construcción & 2 & $1,97 \%$ \\
Inversiones e Inmobiliaria & 16 & $5,18 \%$ \\
Metalmecanicas & 2 & $10,59 \%$ \\
Productos Diversos & 2 & $24,85 \%$ \\
Productos Químicos & 1 & $1,67 \%$ \\
Servicios Públicos & 1 & $0,00 \%$ \\
Textiles y Vestuario & 2 & $0,01 \%$ \\
\hline Total & 36 & $6,77 \%$ \\
\hline
\end{tabular}

\section{Desarrollo en el largo plazo}

\subsection{Resultados de CAR mediante el uso de una cartera temporal}

Las Tablas 4 y 5 muestran los Retornos Ajustados Promedio (AR) y los Retornos Ajustados Acumulados (CAR) de los primeros 36 meses luego del IPO, excluyendo los retornos iniciales para las 35 empresas para las cuales hay precios de largo plazo. En la Tabla 4, los retornos mensuales de la muestra están ajustados por el IGPA y en la Tabla 5 están ajustados por el IPSA. En ambos casos 20 de los 36 AR son negativos.

Para probar el significado económico y estadístico de retornos anormales negativos, se utilizó la variante propuesta por Ritter (1991), que consiste en:

- El estadístico $t$ del retorno ajustado promedio se calcula para cada mes como: $\mathrm{AR}_{t} \times \frac{\sqrt{n_{t}}}{S D_{t}}$, donde $S D_{t}$ es la desviación estándar de los retornos ajustados del mes $t$.

20 El análisis de Ritter (1991) incluye 1203 empresas para el periodo 1975 a 1984.

21 Es importante considerar el reducido tamaño de la muestra, además de haber pocas empresas en cada sector y que pueden no ser representativas, a excepción del sector Inversiones e Inmobiliaria. 
Tabla 4. Retornos ajustados por el IGPA en el largo plazo (3 años), retorno promedio ajustado $\left(A R_{t}\right)$ y retorno ajustado acumulado $\left(\mathrm{CAR}_{0, t}\right)$ con sus estadísticos $t$ correspondiente para los 36 meses después del IPO.

\begin{tabular}{|c|c|c|c|c|c|}
\hline Mes & $\begin{array}{c}\text { Cantidad de } \\
\text { Firmas }\end{array}$ & $\overline{\mathrm{AR}_{t}}$ & $t$-stat & $\overline{\mathrm{CA}} \overline{\mathrm{R}}_{0, t}$ & $t$-stat \\
\hline 1 & 35 & $1.13 \%$ & 0,50 & $1,13 \%$ & 0,80 \\
\hline 2 & 35 & $-1.21 \%$ & $-0,71$ & $-0,07 \%$ & $-0,04$ \\
\hline 3 & 35 & $3,76 \%$ & 1,55 & $3,68 \%$ & 1,52 \\
\hline 4 & 35 & $0,68 \%$ & 0,32 & $4,36 \%$ & 1,56 \\
\hline 5 & 35 & $-1,71 \%$ & $-1,03$ & $2,66 \%$ & 0,85 \\
\hline 6 & 35 & $-3,60 \%$ & $-2,59^{* *}$ & $-0,95 \%$ & $-0,28$ \\
\hline 7 & 35 & $0,85 \%$ & 0,42 & $-0,09 \%$ & $-0,03$ \\
\hline 8 & 35 & $1,97 \%$ & 0,98 & $1,88 \%$ & 0,48 \\
\hline 9 & 35 & $2,60 \%$ & 1,56 & $4,47 \%$ & 1,07 \\
\hline 10 & 35 & $-3,37 \%$ & $-2,22^{* *}$ & $1,11 \%$ & 0,25 \\
\hline 11 & 35 & $-2.59 \%$ & $-2,15^{* *}$ & $-1,48 \%$ & $-0,32$ \\
\hline 12 & 35 & $-0.79 \%$ & $-0,55$ & $-2,27 \%$ & $-0,47$ \\
\hline 13 & 35 & $1,20 \%$ & 0,67 & $-1,07 \%$ & $-0,21$ \\
\hline 14 & 35 & $-4,67 \%$ & $-3,03^{* * *}$ & $-5,74 \%$ & $-1,10$ \\
\hline 15 & 35 & $-0,56 \%$ & $-0,31$ & $-6,30 \%$ & $-1,17$ \\
\hline 16 & 35 & $-1,90 \%$ & $-1,18$ & $-8,20 \%$ & $-1,48$ \\
\hline 17 & 35 & $-2,96 \%$ & $-0,99$ & $-11,16 \%$ & $-1,95^{*}$ \\
\hline 18 & 35 & $1,11 \%$ & 0,46 & $-10,05 \%$ & $-1,71^{*}$ \\
\hline 19 & 35 & $-1,65 \%$ & $-1,05$ & $-11,70 \%$ & $-1,93^{*}$ \\
\hline 20 & 35 & $-3,17 \%$ & $-1,83^{*}$ & $-14,87 \%$ & $-2,39^{* *}$ \\
\hline 21 & 35 & $-1,43 \%$ & $-0,80$ & $-16,30 \%$ & $-2,56^{* *}$ \\
\hline 22 & 35 & $1,32 \%$ & 0,45 & $-14,98 \%$ & $-2,30^{* *}$ \\
\hline 23 & 35 & $0,74 \%$ & 0,34 & $-14,24 \%$ & $-2,14^{* *}$ \\
\hline 24 & 35 & $0,09 \%$ & 0,05 & $-14,15 \%$ & $-2,08^{* *}$ \\
\hline 25 & 35 & $11,18 \%$ & 1,25 & $-2,97 \%$ & $-0,43$ \\
\hline 26 & 35 & $-2,56 \%$ & $-1,77^{*}$ & $-5,53 \%$ & $-0,78$ \\
\hline 27 & 35 & $-3,57 \%$ & $-2,40^{* *}$ & $-9,11 \%$ & $-1,26$ \\
\hline 28 & 35 & $2,30 \%$ & 1,45 & $-6,80 \%$ & $-0,93$ \\
\hline 29 & 35 & $-1,19 \%$ & $-0,60$ & $-7,99 \%$ & $-1,07$ \\
\hline 30 & 35 & $1,84 \%$ & 0,65 & $-6,15 \%$ & $-0,81$ \\
\hline 31 & 35 & $-3,25 \%$ & $-2,03^{*}$ & $-9,40 \%$ & $-1,22$ \\
\hline 32 & 35 & $-0,04 \%$ & $-0,02$ & $-9,44 \%$ & $-1,20$ \\
\hline 33 & 34 & $-2,50 \%$ & $-1,45$ & $-11,94 \%$ & $-1,47$ \\
\hline 34 & 34 & $2,83 \%$ & 1,13 & $-9,10 \%$ & $-1,11$ \\
\hline 35 & 34 & $-2,83 \%$ & $-1,78^{*}$ & $-11,48 \%$ & $-1,38$ \\
\hline 36 & 34 & $2,20 \%$ & $1,98^{*}$ & $-9,29 \%$ & $-1,10$ \\
\hline
\end{tabular}

Nota: Los asteriscos indican si el coeficiente es significativamente distinto de cero al $10 \%, 5 \%$ y $1 \%$. 
Tabla 5. Retornos ajustados por el IPSA en el largo plazo (3 años), retorno promedio ajustado $\left(\mathrm{AR}_{t}\right)$ y retorno ajustado acumulado $\left(\mathrm{CAR}_{0, t}\right)$ con sus estadísticos $t$ correspondientes para los 36 meses después del IPO.

\begin{tabular}{|c|c|c|c|c|c|}
\hline Mes & $\begin{array}{c}\text { Cantidad de } \\
\text { Firmas }\end{array}$ & $\mathrm{AR}_{t}$ & $t$-stat & $\mathrm{CAR}_{0, t}$ & $t$-stat \\
\hline 1 & 35 & $1,25 \%$ & 0,52 & $1.25 \%$ & 0,85 \\
\hline 2 & 35 & $-1,41 \%$ & $-0,80$ & $-0,16 \%$ & $-0,08$ \\
\hline 3 & 35 & $4,24 \%$ & 1,50 & $4,08 \%$ & 1,63 \\
\hline 4 & 35 & $1,17 \%$ & 0,51 & $5,26 \%$ & $1,82^{*}$ \\
\hline 5 & 35 & $-2,89 \%$ & $-1,53$ & $2,37 \%$ & 0,74 \\
\hline 6 & 35 & $-4,59 \%$ & $-3,13^{* * *}$ & $-2,22 \%$ & $-0,63$ \\
\hline 7 & 35 & $0,17 \%$ & 0,08 & $-2,06 \%$ & $-0,54$ \\
\hline 8 & 35 & $2,41 \%$ & 1,17 & $0,35 \%$ & 0,09 \\
\hline 9 & 35 & $2,36 \%$ & 1,25 & $2,71 \%$ & 0,63 \\
\hline 10 & 35 & $-3,84 \%$ & $-2,23^{* *}$ & $-1,12 \%$ & $-0,25$ \\
\hline 11 & 35 & $-2,49 \%$ & $-1,84^{*}$ & $-3,62 \%$ & $-0,76$ \\
\hline 12 & 35 & $-0,48 \%$ & $-0,30$ & $-4,10 \%$ & $-0,82$ \\
\hline 13 & 35 & $0,61 \%$ & 0,34 & $-3,49 \%$ & $-0,67$ \\
\hline 14 & 35 & $-5,20 \%$ & $-2,92^{* * *}$ & $-8,69 \%$ & $-1,62$ \\
\hline 15 & 35 & $0,06 \%$ & 0,03 & $-8,62 \%$ & $-1,55$ \\
\hline 16 & 35 & $-1,85 \%$ & $-1,14$ & $-10,48 \%$ & $-1,82^{*}$ \\
\hline 17 & 35 & $-3,19 \%$ & $-0,99$ & $-13,67 \%$ & $-2,31^{* *}$ \\
\hline 18 & 35 & $1,07 \%$ & 0,45 & $-12,59 \%$ & $-2,07^{* *}$ \\
\hline 19 & 35 & $-2,62 \%$ & $-1,54$ & $-15,21 \%$ & $-2,43^{* *}$ \\
\hline 20 & 35 & $-3,40 \%$ & $-1,91^{*}$ & $-18,61 \%$ & $-2,90^{* * *}$ \\
\hline 21 & 35 & $-2,07 \%$ & $-1,13$ & $-20,69 \%$ & $-3,14^{* * *}$ \\
\hline 22 & 35 & $1,81 \%$ & 0,60 & $-18,88 \%$ & $-2,80^{* * *}$ \\
\hline 23 & 35 & $0,82 \%$ & 0,34 & $-18,06 \%$ & $-2,62^{* *}$ \\
\hline 24 & 35 & $-0,07 \%$ & $-0,03$ & $-18,12 \%$ & $-2,58^{* *}$ \\
\hline 25 & 35 & $10,13 \%$ & 1,15 & $-7,99 \%$ & $-1,11$ \\
\hline 26 & 35 & $-3,84 \%$ & $-2,49^{* *}$ & $-11,83 \%$ & $-1,62$ \\
\hline 27 & 35 & $-4,25 \%$ & $-2,62^{* *}$ & $-16,08 \%$ & $-2,16^{* *}$ \\
\hline 28 & 35 & $2,68 \%$ & 1,60 & $-13,40 \%$ & $-1,76^{*}$ \\
\hline 29 & 35 & $-1,61 \%$ & $-0,68$ & $-15,01 \%$ & $-1,94^{*}$ \\
\hline 30 & 35 & $1,67 \%$ & 0,58 & $-13,34 \%$ & $-1,70^{*}$ \\
\hline 31 & 35 & $-4,34 \%$ & $-2,63^{*}$ & $-17,69 \%$ & $-2,21^{* *}$ \\
\hline 32 & 35 & $-0,18 \%$ & $-0,10$ & $-17,87 \%$ & $-2,20^{* *}$ \\
\hline 33 & 34 & $-3,20 \%$ & $-1,83^{*}$ & $-21,07 \%$ & $-2,52^{* *}$ \\
\hline 34 & 34 & $2,06 \%$ & 0,76 & $-19,01 \%$ & $-2,24^{* *}$ \\
\hline 35 & 34 & $-2,76 \%$ & $-1,82^{*}$ & $-21,77 \%$ & $-2,53^{* *}$ \\
\hline 36 & 34 & $0,99 \%$ & 0,77 & $-20,79 \%$ & $-2,38^{* *}$ \\
\hline
\end{tabular}

Nota: Los asteriscos indican si el coeficiente es significativamente distinto de cero al $10 \%, 5 \%$ y $1 \%$. 
- Siguiendo a Ritter (1991) se obtuvo el estadístico $t$ del CAR como: $\mathrm{CAR}_{1, t} \times \frac{\sqrt{n_{t}}}{C S D_{t}}$ y $C S D_{t}=[t \times \mathrm{var}+2(t-1) \times \operatorname{cov}]^{1 / 2}$.

Donde "var" es el promedio de la varianza de AR sobre los 36 meses y "cov" es la autocovarianza de orden 1 de la serie AR para la muestra en estudio. Para los retornos mensuales ajustados por el IGPA de la Tabla 4, "var" toma un valor de 0.007 y "cov" un valor de -0.0001311 . Por otra parte, para el caso de los retornos mensuales ajustados por el IPSA de la Tabla 5, "var" toma un valor de 0.008 y "cov" un valor de -0.0001504 .

De esta forma, para el caso de los retornos mensuales ajustados por el IGPA sólo 5 de los 20 AR negativos tienen estadísticos $t$ significativos al $5 \%$. Esta situación se profundiza para los retornos mensuales ajustados por el IPSA, ya que 6 de los $20 \mathrm{AR}$ negativos tienen estadísticos $t$ significativos al $5 \%$.

Los CAR fluctúan durante el primer año independiente del índice de mercado utilizado como benchmark. Sin embargo, a partir del mes 12 tienen una tendencia decreciente que no es sistemática, a diferencia de lo reportado en Ritter (1991), ya que tienden a estabilizarse en torno al $-10 \%$ para el caso de los retornos anormales acumulados ajustados por el IGPA y en torno al -15\% para los CAR ajustados por el IPSA. Por otro lado, estos últimos retoman la tendencia decreciente a partir del mes 30. Los CAR son significativamente menores que cero al $5 \%$ solamente entre los meses 20 y 24 cuando se utiliza como benchmark el IGPA. Cuando se utiliza como benchmark el IPSA, los CAR son significativamente menores que cero al $5 \%$ entre los meses 17 y 24 y se vuelven de manera sistemática estadísticamente menores que cero al $5 \%$ a partir del mes 31.

Por lo tanto, el bajo desempeño a largo plazo de las IPO's resulta económica y estadísticamente significativo, aunque estos son evidentemente más severos cuando se utiliza como benchmark el IPSA.

La Figura 2 muestra lo diferentes que resultan los CAR excluyendo el retorno inicial cuando se utiliza como benchmark el IGPA e IPSA,${ }^{22}$ pudiéndose observar lo severo que resultan los CAR durante el último año cuando se utiliza como grupo de control el IPSA.

En la Figura 3 es posible apreciar los retornos anormales acumulados diarios, ${ }^{23}$ utilizando el IGPA como grupo de control, los retornos crudos acumulados diarios (RAW) ${ }^{24}$ y los retornos acumulados del benchmark, para los cuales se utilizó el IGPA.

Es posible apreciar que tanto el RAW como el IGPA muestran un comportamiento similar, es decir fluctúan hasta el primer año y luego ambos reportan una tendencia decreciente. Dicha situación difiere con la Figura 4, la cual muestra los retornos anormales acumulados diarios utilizando el IPSA como grupo de control, los retornos crudos acumulados diarios (RAW) y los retornos acumulados del benchmark, para los cuales esta vez se utilizó el IPSA.

22 Las series de CAR corresponden a las obtenidas de las Tabla 4 y Tabla 5.

23 A diferencia de los análisis anteriores, esta vez se utilizó el retorno real diario y no el mensual de las IPO's, como del benchmark, para el cálculo de los AR y de los CAR.

24 Los retornos crudos acumulados corresponden a los que no han sido ajustados por un grupo de control o benchmark. 
Figura 2. Retornos ajustados acumulados(CAR):

ajustados por el IGPA y el IPSA

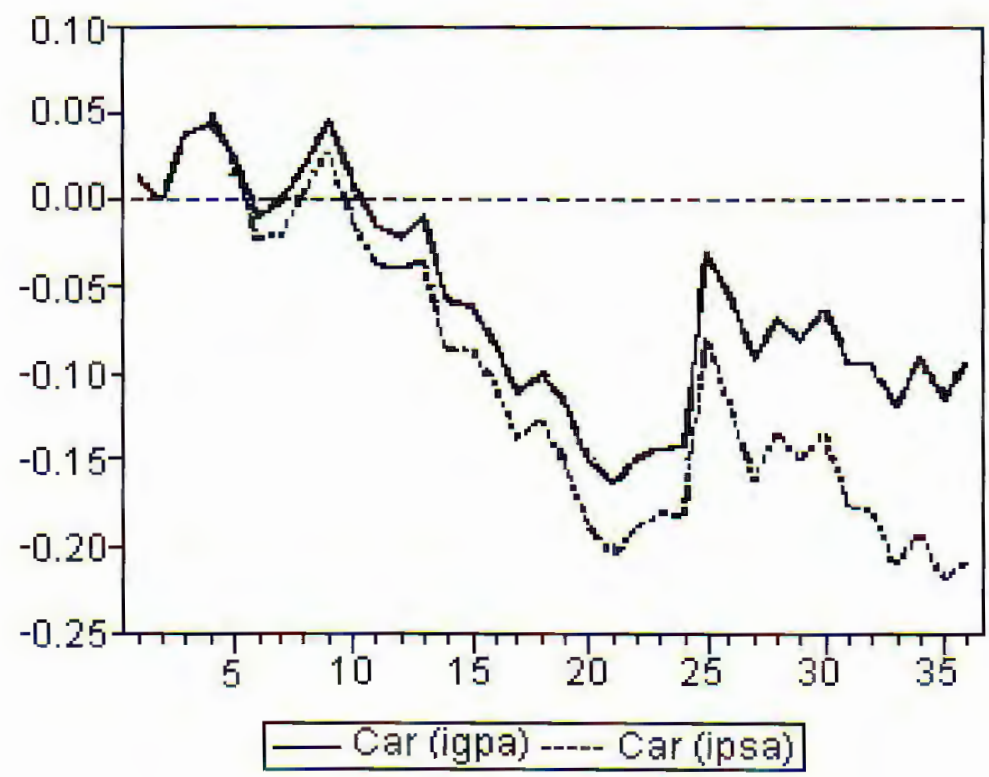

Figura 3. Retornos ajustados acumulados (CAR): ajustado por el IGPA, retornos crudos acumulados diarios (RAW) y retornos acumulados (CR) del IGPA

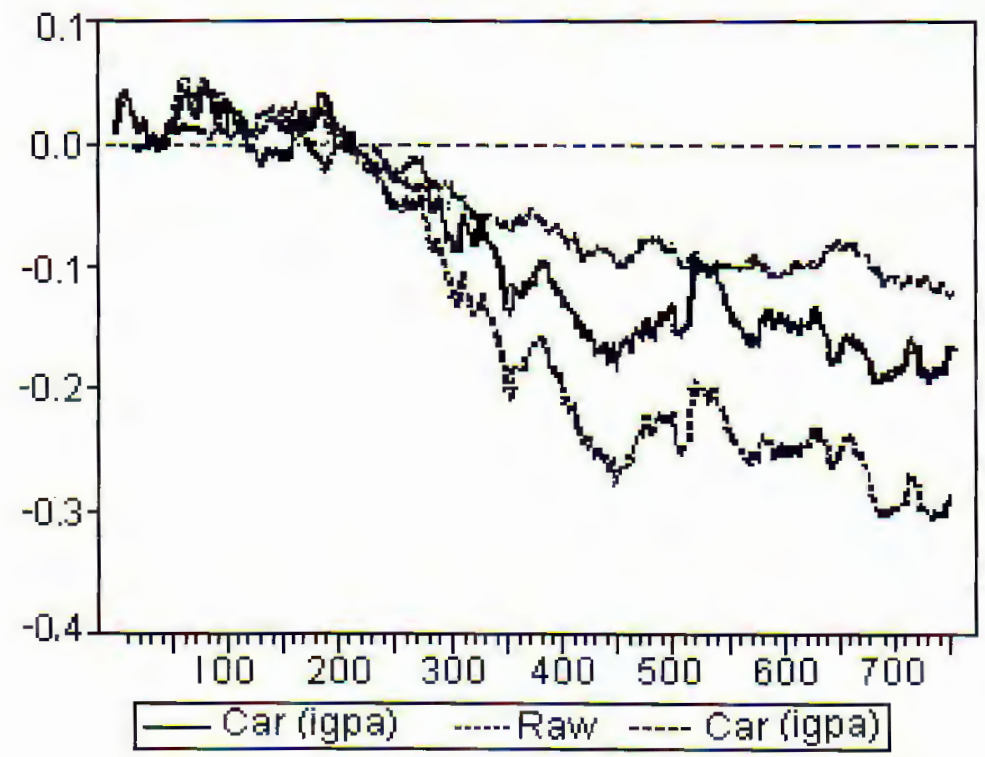


Figura 4. Retornos ajustados acumulados (CAR): ajustado por el IPSA, retornos crudos acumulados diarios (RAW) y retornos acumulados $(\mathrm{CR})$ del IPSA

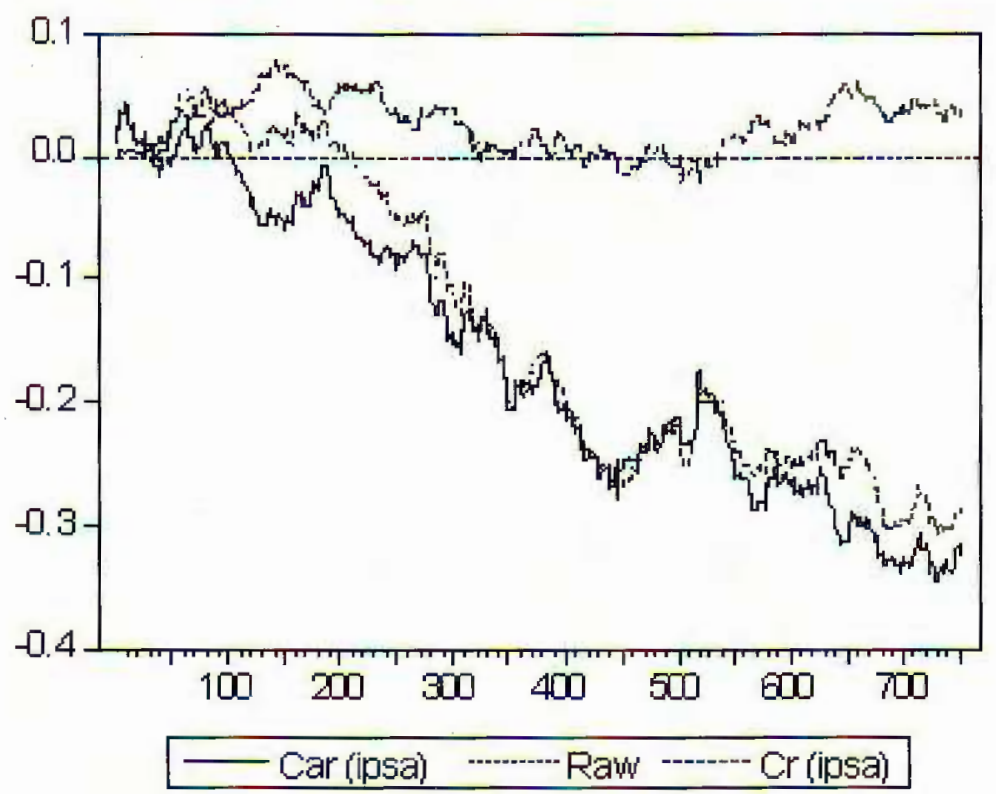

El retorno acumulado del IPSA no muestra la tendencia decreciente que se aprecia para los retornos acunulados del IGPA, fluctuando en torno al $0 \%$ durante el periodo de estudio. Esta situación explica las diferencias entre los CAR, al escoger diferentes grupos de control como benchmark.

Utilizando como benchmark el Índice de Precios Selectivo de Acciones (IPSA), ${ }^{25}$ cuya finalidad es reflejar las variaciones de precio de los títulos más activos del mercado, no resulta ser un buen grupo de control para inferir sobre la existencia de un bajo desempeño a largo plazo de las IPO's, ya que la Ofertas Públicas Iniciales son en su mayoría de empresas pequeñas, es decir, de bajo patrimonio bursátil. Al analizar la distribución de los Retornos Reales diarios del IPSA e IGPA para el periodo analizado se descubre que, si bien ambos índices se distribuyen de manera similar, los retornos reales diarios del IPSA reportan una varianza mayor a la de los retornos reales diarios del IGPA así como un retorno promedio mayor. Dada esta situación es posible apreciar un sesgo positivo de los retornos diarios del IPSA, producto de que dicho índice incorpora empresas de mayor tamaño.

Para evitar dicho sesgo, resulta conveniente utilizar el Índice General de Precios de Acciones (IGPA), ya que ante la imposibilidad de construir un grupo de control conformado por empresas similares, ${ }^{26}$ el cual agrupa a casi la totalidad de las acciones con cotización bursátil.

25 Considera las 40 acciones con mayor presencia bursátil.

26

En tarnaño y razón $\mathrm{BE} / \mathrm{ME}$. 


\subsection{Resultados de retornos por tenencia en el largo plazo (HPR)}

Los retornos más altos luego de 3 años corresponden a Santa Isabel (285.72\%), D\&S (212.41\%) y Rebrisa-A (186.80\%), excluyendo del cálculo el retorno inicial del día de colocación. La mayoría de las IPO's muestran un bajo desempeño, donde el histograma de la Figura 5 indica que su media se ubica alrededor del $-9.5 \%$. En el mismo periodo el índice IGPA promedió $-10.2 \%$, resultando ser el índice de riqueza relativa al 0.99 para las 35 empresas con datos de largo plazo.

Las Figuras 5 y 6 muestran la distribución de los retornos a 3 años de las IPO's y del IGPA en el mismo periodo. Los retornos de las IPO's tienen una menor dispersión y simetría.

Figura 5. Distribución de los HPR de las IPO's en los primeros 3 años

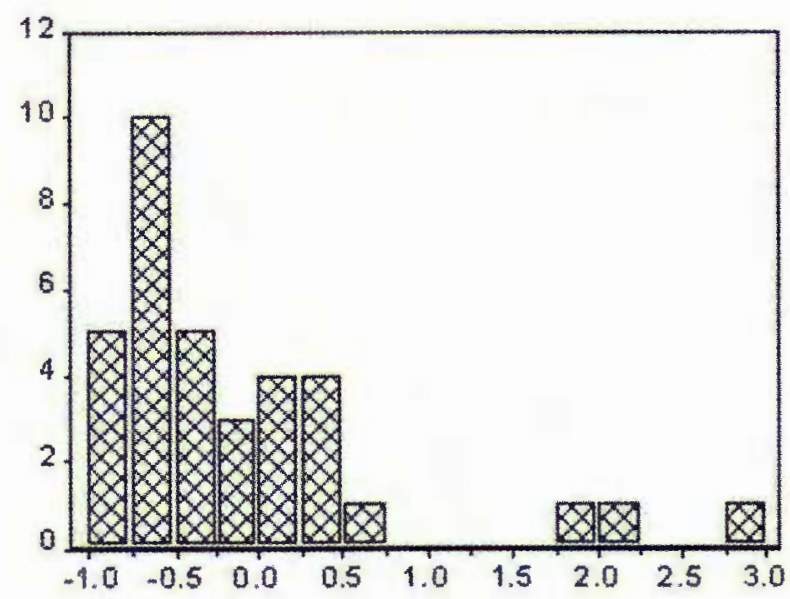

\begin{tabular}{|c|c|}
\hline \multicolumn{2}{|c|}{$\begin{array}{l}\text { Series HPR IPO } \\
\text { Sarqle } 135 \\
\text { Obsavatons } 35\end{array}$} \\
\hline Mean & .0095704 \\
\hline Wedian & .0335276 \\
\hline Mexinum & 2857243 \\
\hline Mrimum & -0.895938 \\
\hline $\begin{array}{l}\text { Std. Der. } \\
\text { Skevers }\end{array}$ & $\begin{array}{l}0.860313 \\
1.833709\end{array}$ \\
\hline Kutosis & 6.529358 \\
\hline sucue Bea & 39.97770 \\
\hline Prokebity & 0.000000 \\
\hline
\end{tabular}

Figura 6. Distribución de los HPR del IGPA en los primeros 3 años

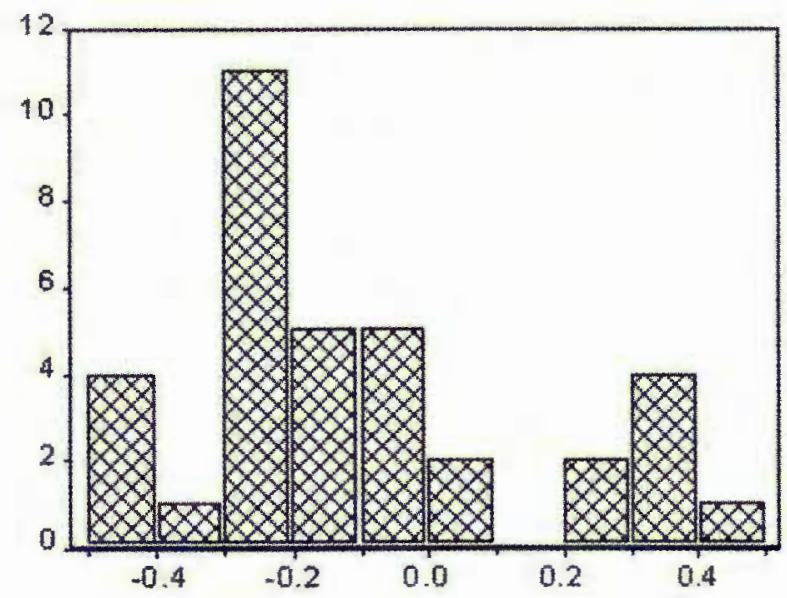

\begin{tabular}{|c|c|}
\hline \multicolumn{2}{|c|}{$\begin{array}{l}\text { Sene HFR igpa } \\
\text { Sample } 135 \\
\text { Chsevatione } 35\end{array}$} \\
\hline mean & -0.101573 \\
\hline Medien & -0.181503 \\
\hline heximun & 0.469112 \\
\hline Mrimun & -0.461741 \\
\hline $\begin{array}{l}\text { Std.Der. } \\
\text { Skevness }\end{array}$ & $\begin{array}{l}0.252805 \\
0.780013\end{array}$ \\
\hline Kutcas & 2.661902 \\
\hline 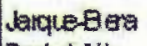 & 3.715819 \\
\hline Prakeblity & 0.155998 \\
\hline
\end{tabular}


Para interpretar estos resultados de largo plazo, la muestra se presenta a través de divisiones por tamaño, retornos iniciales, sector, año de emisión y underwriter. De este modo se busca reconocer ciertas características de la emisión inicial que puedan ser causa de retornos anormales.

Muchos estudios reportan que las emisiones más pequeñas tienen mayores retornos iniciales y peor desempeño de largo plazo, lo que se interpreta como un aprovechamiento de empresas pequeñas y riesgosas del clima positivo de los inversionistas. La Tabla 6 muestra un patrón similar: las empresas que emiten inicialmente montos pequeños presentan mayores retornos iniciales y un bajo desempeño a largo plazo $(\mathrm{WR}<1)$.

La mayoría de las colocaciones durante el primer día fluctuaron entre $\$ 1.507 .690$ y $\$ 3.775 .787 .835$, concentrado en 26 de las 35 IPO's consideradas para este análisis.

Tabla 6. Retornos iniciales y de largo plazo ordenados por tamaño de la emisión

\begin{tabular}{|cccccc|}
\hline & \multirow{2}{*}{ Retornos } & \multicolumn{3}{c|}{ Retorno a 3 años } & \\
\cline { 3 - 5 } Monto Emitido $(\$)$ & Iniciales & \multicolumn{1}{c|}{ IPO } & IGPA & WR & $N^{o}$ \\
\hline $1.507 .690,0-3.775 .787 .835,0$ & $9,08 \%$ & $-10,08 \%$ & $-6,14 \%$ & 0,95 & 26 \\
$3.775 .787 .835,0-7.550 .067 .980,1$ & $0,00 \%$ & $-82,31 \%$ & $-32,16 \%$ & 0,26 & 2 \\
$7.550 .067 .980,1-11.324 .348 .125,1$ & $0,00 \%$ & $-19,72 \%$ & $-17,69 \%$ & 0,98 & 1 \\
$11.324 .348 .125,1-15.098 .628 .270,2$ & $3,76 \%$ & $-59,59 \%$ & $-19,24 \%$ & 0,51 & 2 \\
$15.098 .628 .270,2-18.872 .908 .415,2$ & $0,00 \%$ & $26,53 \%$ & $-25,73 \%$ & 1,70 & 1 \\
$18.872 .908 .415,2-22.647 .188 .560,3$ & $0,00 \%$ & $68,07 \%$ & $-16,58 \%$ & 1,91 & 3 \\
\hline Total & $6,96 \%$ & $-9,57 \%$ & $-10,16 \%$ & 0,99 & 35 \\
\hline
\end{tabular}

Tabla 7. Desempeño de largo plazo ordenada por retornos iniciales

\begin{tabular}{|ccccc|}
\hline & \multicolumn{3}{c}{ Retorno a 3 años } \\
\cline { 2 - 4 } Retornos Iniciales & IPO & IGPA & WR & N \\
\hline$-26,48 \%<\mathrm{RI}<-14,51 \%$ & $-60,80 \%$ & $-21,72 \%$ & 0,50 & 1 \\
$-14,51 \%<\mathrm{RI}<-2,54 \%$ & $-63,44 \%$ & $-21,72 \%$ & 0,47 & 2 \\
$-2,54 \%<\mathrm{RI}<9,43 \%$ & $1,21 \%$ & $-4,01 \%$ & 1,07 & 23 \\
$9,43 \%<\mathrm{RI}<21,41 \%$ & $-1,47 \%$ & $-20,24 \%$ & 1,04 & 4 \\
$21,41 \%<\mathrm{RI}<33,38 \%$ & $-8,27 \%$ & $-37,86 \%$ & 1,48 & 1 \\
$33,38 \%<\mathrm{RI}<45,35 \%$ & $-40,25 \%$ & $-19,82 \%$ & 0,75 & 4 \\
\hline Total & $-9,57 \%$ & $-10,16 \%$ & 0,99 & 35 \\
\hline
\end{tabular}

A grandes rasgos parecería que a mayor underpricing, mejor desempeño a largo plazo, según lo constatado en la Tabla 7. Sin embargo, esta relación es poco clara. En la Tabla 8 se aprecian los resultados de la regresión:

$$
\mathrm{WR}=0.97+0.24 \times \text { underpricing } .
$$


Donde el parámetro de la variable underpricing resultó ser positivo y significativo. Sin embargo, la regresión arrojo un $R^{2}$ demasiado bajo (0.001). De esta manera, se constató que no existe ninguna asociación lineal entre el underpricing y el indice de riqueza (WR), por lo que pretender ajustar dicha causalidad por una recta no resulta ser significativo. Así, la Tabla 7 sólo establece que una cartera equiponderada de IPO's con retornos iniciales cercanos al promedio encontrado (6.77\%), tuvieron un buen desempeño en el largo plazo (WR > 1), no así la cartera equiponderada de IPO's con retornos iniciales más extremos.

En este caso el underpricing no parece representar un over reaction, sino que es parte de un ajuste parcial que se completa con ajustes de precios en años posteriores. Además, en este análisis parece no haber ninguna relación económica ni estadística entre el underpricing y el desempeño de largo plazo. Lo anterior es compatible con un mercado menos desarrollado que el mercado americano donde se realizó el estudio de Ritter (1991).

Tabla 8. Regresión de desempeño de largo plazo (WR) y underpricing

\begin{tabular}{lcccc}
\hline $\begin{array}{l}\text { Dependent Variable: WR } \\
\text { Method: Least Squares }\end{array}$ & & & \\
Sample: 135 & & & & \\
Included observations: 35 & & & & \\
\hline Variable & Coefficient & Std. Error & t-Statistic & Prob. \\
\hline Underpricing & 0.239170 & 0.930885 & 0.256928 & 0.7988 \\
C & 0.974738 & 0.155745 & 6.258528 & 0.0000 \\
\hline R-squared & 0.001996 & & & \\
Adjusted R-squared & -0.028246 & & & \\
\hline
\end{tabular}

En general, casi todos los sectores económicos presentaron un bajo desempeño de largo plazo, dado que reportaban $\mathrm{WR}<1$, con la excepción de los sectores Comerciales y Distribuidores, Metalmecánica, Servicios Públicos y Construcción. El sector que concentra el mayor número de IPO's (Inversiones e Inmobiliarias con 15) presenta un bajo desempeño a largo plazo, constatándose un WR $<1(0.70)$. Estas diferencias entre los diferentes sectores se pueden interpretar como:

- Optimismo irracional de los inversionistas, dirigido hacia ciertas industrias en particular.

- Consecuencia de no ajustar utilizando los "betas" de cada industria.

- Producto de la escasa cantidad de empresas en algunos sectores de la economía.

La interpretación de optimismo de los inversionistas que emplea Ritter (1991) se ve respaldada en una muestra de 1526 IPO's en Estados Unidos. Sin embargo, la muestra empleada en esta investigación contiene sólo 35 empresas.

Por otro lado, tanto los retornos iniciales como el desempeño de largo plazo varían mucho entre los diferentes años de emisión. Por ejemplo, las emisiones lanzadas en 1996 tuvieron un buen desempeño en el largo plazo. Sin embargo, 
esta investigación no cuenta con una muestra idealmente representativa para concluir a este respecto.

\subsection{Desempeño de largo plazo por banco Underwriter}

Los Bancos que actúan de underwriter tienen incentivos a realizar la oferta al mayor precio posible para reducir el costo de financiamiento del emisor y, por otro lado, a realizar la oferta a un precio bajo, para dar un retorno aceptable a los inversionistas, preservando de esa manera su reputación como colocador de acciones.

A causa de la asimetría de información, los underwriters se deben construir una buena reputación, estableciendo un precio razonable según las proyecciones a largo plazo de la empresa, quizás sin aprovechar al máximo el optimismo "irracional" de los mercados y dejando grandes retornos iniciales.

Sin embargo, en el largo plazo las acciones emitidas por un banco, que se preocupa por su reputación, van a ofrecer retornos similares o mayores a los que ofrecen empresas comparables, pero ciertamente no menores. En la Tabla 9 se aprecia que todos los underwriters que participaron de las colocaciones de esta investigación, reportan en promedio retornos iniciales positivos, no tanto así en el largo plazo. Sin embargo, CITICORP y IM TRUST presentan retornos iniciales elevados y las acciones colocadas por ellos poseen un alto desempeño en el largo plazo, concluyendo que estos dos underwriters se han forjado una muy buena reputación entre los agentes que participan del Mercado de Valores. ${ }^{27}$

Tabla 9. Desempeño por banco underwriter

\begin{tabular}{|crrrrrr|}
\hline & Retorno & \multicolumn{3}{c|}{ Retorno a 3 años } & \\
\cline { 3 - 5 } UNDERWRITER & Inicial & \multicolumn{1}{c|}{ IPO } & \multicolumn{1}{c|}{ IGPA } & WR & No \\
\hline BANKERS & $1,25 \%$ & $-32,46 \%$ & $-26,27 \%$ & 0,88 & 6 \\
BICE & $0,00 \%$ & $-20,20 \%$ & $9,76 \%$ & 0,86 & 2 \\
CITICORP & $24,25 \%$ & $-7,05 \%$ & $2,48 \%$ & 0,96 & 3 \\
IM TRUST & $42,43 \%$ & $9,89 \%$ & $-9,67 \%$ & 1,22 & 1 \\
LARRAIN & $3,26 \%$ & $4,36 \%$ & $-1,48 \%$ & 1,17 & 8 \\
NEVASA & $2,29 \%$ & $-47,72 \%$ & $34,10 \%$ & 0,39 & 1 \\
SANTANDER & $0,00 \%$ & $-19,72 \%$ & $-17,69 \%$ & 0,98 & 1 \\
SECURITY & $0,62 \%$ & $55,94 \%$ & $-7,16 \%$ & 1,68 & 1 \\
TANNER & $0,15 \%$ & $-89,59 \%$ & $-25,26 \%$ & 0,14 & 1 \\
OTRO & $7,64 \%$ & $0,11 \%$ & $-16,88 \%$ & 1,04 & 12 \\
\hline
\end{tabular}

Algunos de los cortes transversales que intentan explicar los retornos de largo plazo no son independientes. Por ejemplo, las emisiones de mayor tamaño y las de altos retornos iniciales, tuvieron en general un buen desempeño en el largo plazo. Pero no se ha establecido en el análisis previo que muchas de las

27 Reputación lograda con un trade off entre colocar dinero sobre la mesa y prometer altos desempeños a largo plazo en las acciones colocadas. 
empresas de gran tamaño también tuvieron altos retornos iniciales. Dado lo anterior se analizan los efectos del underpricing sobre la estrategia de comprar la acción al cierre del primer día y venderla a los tres años o al momento en que se retira de cotización.

La. Tabla 10 presenta los resultados de la regresión estimada por mínimos cuadrados ordinarios para explicar el retorno de largo plazo en función del retorno inicial y del retorno de mercado.

$$
\mathrm{HPR}=-0.0058+0.2733 \times \text { underpricing }+1.0717 \times \mathrm{HPR}_{\mathrm{IGPA}}
$$

Tabla 10. Regresión por OLS para explicar los retornos de largo plazo HPR (IPO) en función de underpricing y HPR (IGPA)

de mercado en un periodo equivalente

\begin{tabular}{|c|c|c|c|c|}
\hline \multicolumn{5}{|c|}{ Dependent Variable: HPR(IPO) } \\
\hline \multicolumn{5}{|c|}{ Method: Least Squares } \\
\hline \multicolumn{5}{|l|}{ Sample:1 35} \\
\hline \multicolumn{5}{|c|}{ Included observations: 35} \\
\hline Variable & Coefficient & Std. Error & $\mathrm{t}$-Statistic & Prob. \\
\hline Underpricing & 0.273353 & 0.946869 & 0.288692 & 0.7747 \\
\hline HPR(igpa) & 1.071738 & 0.578175 & 1.853656 & 0.0730 \\
\hline $\mathrm{C}$ & -0.005870 & 0.164085 & -0.035772 & 0.9717 \\
\hline $\mathrm{R}$-squared & 0.096968 & & & \\
\hline Adjusted R-squared & 0.040529 & & & \\
\hline
\end{tabular}

La variable underpricing tiene un coeficiente positivo, aunque no significativo a los niveles usuales y el retorno del mercado no permite explicar el retorno de una IPO durante los primeros 3 años. Muchos modelos de valoración de activos, como el CAPM, predicen que el mercado sólo pagará un mayor retorno por el riesgo sistemático de un activo y no por otros riesgos intrínsecos al modelo, ya que estos debieran ser diversificables. De este modo, uno esperaría que el coeficiente de mercado fuera significativo.

\subsection{Resultados utilizando el Modelo de Tres Factores de Fama y French $(1992,1993)$}

En la utilización del modelo de tres factores de Fama y French, para cada mes del periodo comprendido desde julio 1993 a diciembre de 2000, se obtuvo la rentabilidad en exceso sobre la tasa libre de riesgo de carteras equiponderadas compuestas por las IPO's de la muestra. ${ }^{28}$

Los factores ${ }^{29}$ se construyeron a partir de la creación de carteras mensuales, según el patrimonio bursátil y la razón valor libro / valor de mercado (BE/ME),

28 Es decir, $\left(R_{p, t}-R_{f, t}\right)$.

29 En el modelo de tres factores de Fama y French, a saber $R_{p, t}-R_{f, t}=\alpha_{p}+\beta_{p}\left(R_{m, t}-\right.$ $\left.R_{f, t}\right)+s_{p}\left(S M B_{t}\right)+h_{p}\left(H M L_{t}\right)+\varepsilon_{p t}$, los tres factores son: $S M B_{t}, H M L_{t}$ y el factor mercado $\left(R_{m, t}-R_{f, t}\right)$. 
de todas las empresas que presentaban acciones transadas cada mes. En esta investigación se optó por la creación de todas las carteras que se explican a continuación, para cada uno de los meses en estudio, permitiendo el rebalanceo mensual de las mismas. De esta forma, se dejó que las empresas pudiesen cambiar de grupo o cartera mes a mes.

Para cada mes durante el periodo comprendido entre 1993 - 2000 se ordenó por patrimonio bursátil las empresas que cotizaron en la Bolsa de Comercio de Santiago. Se obtuvo, tanto la rentabilidad de una cartera conformada por las empresas que presentaron un patrimonio bursátil por debajo del promedio (SMALL), así como la de una cartera conformada por las empresas que presentaron un patrimonio bursátil por sobre el promedio (BIG). $S M B_{t}$ se obtuvo a partir de la diferencia entre el promedio de los retornos SMALL y el promedio de los retornos de las acciones BIG.

Posteriormente, se obtuvo la rentabilidad mensual de 4 carteras equiponderadas requeridas para la construcción del factor $H M L$. Estas son:

- BIG HIGH (BH). Cartera conformada por empresas que poseen un patrimonio bursátil por sobre el promedio del total de acciones que cotizaron durante el mes $i$ y que de este subgrupo se encuentran entre el percentil 70 y 100 de los mayores valores libro/bolsa. ${ }^{30}$

- BIGH LOW (BL). Cartera conformada por empresas que poseen un patrimonio bursátil por sobre el promedio del total de acciones que cotizaron durante el mes $i$ y que de este subgrupo se encuentran entre el percentil 0 y 30 de los mayores valores libro/bolsa.

- SMALL HIGH (SH). Cartera conformada por empresas que poseen un patrimonio bursátil por debajo del promedio del total de acciones que cotizaron durante el mes $i$ y que de este subgrupo se encuentran entre el percentil $70 \mathrm{y}$ 100 de los mayores valores libro/bolsa.

- SMALL LOW (SL). Cartera conformada por empresas que poseen un patrimonio bursátil por debajo del promedio del total de acciones que cotizaron durante el mes $i$ y que de este subgrupo se encuentran entre el percentil 0 y 30 de los mayores valores libro/bolsa.

De esta forma el factor $H M L$ se obtuvo a partir de la diferencia entre los promedios de los retornos de las carteras HIGH (BH y SH) y LOW (BL y SL). La tasa de mercado fue el IGPA, la misma utilizada como benchmark en los modelos anteriores. Ya que al ser el IPSA un índice selectivo, no es idóneo para representar al mercado. De esta forma el factor MERCADO se construyó a partir de la rentabilidad en exceso sobre la tasa libre de riesgo del IGPA, mes a mes.

Se define como la tasa libre de riesgo, la de los instrumentos de inversión de corto plazo que ofrece el banco central (T-BILL ${ }^{31}$ de E.E.U.U.). Por esta

30 Que es la relación inversa del valor bolsa/valor libro que aparece en los informativos mensuales de la Bolsa de Comercio de Santiago.

31 Pagaré del tesoro americano de corto plazo. Este instrumento es definido en la literatura como de cero riesgo. 
razón, se ha considerado que $\operatorname{los} \mathrm{PRBC}^{32}$ a 90 días reflejan más acabadamente esta definición para el mercado nacional.

Antes de utilizar el modelo de tres factores de Fama y French (1993), que depura el grupo de control entre empresas de diferentes tamaños y razones $\mathrm{BE} / \mathrm{ME}$, se utilizó el modelo de CAPM (puesto que en el presente estudio se consideró al IGPA como benchmark).

Los resultados del modelo de CAPM se muestran en la Tabla 11. En la cual se constató la "existencia" de retornos anormales a largo plazo, ya que la constante toma un coeficiente negativo que resulta ser estadística y económicamente significativo al $95 \%$ de confianza.

Tabla 11. Regresión por OLS para aplicar la prueba a la constante y determinar retornos anormales mediante CAPM

(julio de 1993 a diciembre de 2000)

\begin{tabular}{lcccc}
\hline $\begin{array}{l}\text { Dependent Variable: Cartera(IPO) } \\
\text { Method: Least Squares } \\
\text { Sample: } 190\end{array}$ & & & \\
Included observations: 90 & & & & \\
\hline Variable & Coefficient & Std. Error & t-Statistic & Prob. \\
\hline MERCADO & 0.760608 & 0.068668 & 11.07664 & 0.0000 \\
C & -0.017699 & 0.005956 & -2.971405 & 0.0038 \\
R-squared & 0.582329 & & & \\
Adjusted R-squared & 0.577583 & & & \\
\hline
\end{tabular}

La variable MERCADO resulta ser un factor relevante que explica el retorno obtenido por una cartera conformada por las IPO's de la muestra. Lo que reafirma la utilización del índice IGPA como grupo de control para constatar retornos anormales.

Fama (1998) y Brav (2000) aducen que la mala evolución de las IPO's se debe a que este es un comportamiento típico de las empresas pequeñas y con una razón $\mathrm{BE} / \mathrm{ME}$ bajo o mediano, grupo en el que según estos autores se concentra este tipo de operaciones. La Tabla 12 muestra la composición de las IPO's en función de las características tamaño y razón BE/ME, a diciembre del año de la oferta.

Tabal 12. Distribución de las IPO's del estudio, según tamaño y razón $\mathrm{BE} / \mathrm{ME}$, a diciembre del año de la oferta

\begin{tabular}{|cccc|}
\hline Razon & \multicolumn{2}{c|}{ Tamaño } & \\
\cline { 2 - 3 } BE/ME & Pequeño & Grande & $N^{o}$ \\
\hline Bajo & 9 & 5 & 14 \\
Mediano & 15 & & 15 \\
Alto & 6 & 1 & 7 \\
\hline Total & 30 & 6 & 36 \\
\hline
\end{tabular}

32 Pagaré reajustable del Banco Central de Chile 
De esta manera, se ha constatado que las IPO's del periodo del presente análisis han sido llevadas a cabo por empresas pequeñas e incipientes con altas expectativas de crecimiento. Los resultados del modelo de tres factores de Fama y French se muestran en la Tabla 13.

Tabla 13. Regresión por OLS para aplicar la prueba a la constante y determinar retornos anormales mediante el modelo de tres factores de Fama y French (julio de 93 a diciembre de 2000)

\begin{tabular}{lcccc}
\hline $\begin{array}{l}\text { Dependent Variable: Cartera (IPO) } \\
\text { Method: Least Squares } \\
\text { Sample: } 190\end{array}$ & & & \\
Included observations: 90 & & & & \\
\hline Variable & Coefficient & Std. Error & t-Statistic & Prob. \\
\hline MERCADO & 0.929913 & 0.073551 & 12.64319 & 0.0000 \\
SMB & 0.325582 & 0.093617 & 3.477823 & 0.0008 \\
HML & 0.388140 & 0.091269 & 4.252694 & 0.0001 \\
C & 0.005809 & 0.006941 & 0.836940 & 0.4049 \\
\hline R-squared & 0.682988 & & & \\
Adjusted R-squared & 0.671929 & & & \\
\hline
\end{tabular}

Para el modelo de tres factores de Fama y French se constató la "inexistencia" de retornos anormales a largo plazo, ya que la constante toma un coeficiente que no resulta ser estadística ni económicamente significativo al $95 \%$ de confianza. La variable MERCADO resulta ser un factor relevante que explica el retorno obtenido por una cartera conformada por las IPO's de la muestra. Además, los factores del modelo ( $S M B$ y $H M L$ ) resultan ser significativos, poniendo de manifiesto que el modelo de tres factores de Fama y French ofrece un resultado confiable. Además posee un $R^{2}$ alta y aceptable para este tipo de modelo.

En la Tabla 14 se realizó una división de la muestra en estudio en función del tamaño de la empresa que llevo a cabo la IPO's, según los mismos criterios utilizados por el modelo de Fama y French. De esta forma, se constató que fueron las empresas pequeñas las que muestran un bajo desempeño a largo plazo (WR < 1), no así las empresas que presentan un patrimonio bursátil superior al promedio en diciembre del año de la oferta.

Tabla 14 Desempeño de largo plazo por tamaño

\begin{tabular}{|cccccc|}
\hline & Retornos & \multicolumn{3}{c|}{ Retornos a 3 años } & \\
\cline { 3 - 5 } Tamaño & Iniciales & IPO & IGPA & WR & $N^{o}$ \\
\hline Grande & $0,00 \%$ & $42,95 \%$ & $-18,70 \%$ & 1,71 & 6 \\
Pequeño & $8,40 \%$ & $-20,44 \%$ & $-8,39 \%$ & 0,84 & 29 \\
\hline Total & $6,96 \%$ & $-9,57 \%$ & $-10,16 \%$ & 0,99 & 35 \\
\hline
\end{tabular}


La división de la muestra en estudio, según la razón $\mathrm{BE} / \mathrm{ME}$ que aparece en la Tabla 15, establece que fueron las empresas con razón $\mathrm{BE} / \mathrm{ME}$ mediano y alto las que presentaron un bajo desempeño en el largo plazo $(\mathrm{WR}<1)$. Las empresas que poseían un negocio inmaduro con altas expectativas de crecimiento (es decir, una razón baja), presentaron un buen desempeño en el largo plazo $(\mathrm{WR}=1.41)$.

Tabla 15. Desempeño de largo plazo por razón BE/ME

\begin{tabular}{|cccccc|}
\hline Razón & Retornos & \multicolumn{3}{c|}{ Retorno a 3 años } & \\
\cline { 3 - 5 } BE/ME & Iniciales & IPO & IGPA & WR & $N^{\circ}$ \\
\hline Bajo & $7,72 \%$ & $35,65 \%$ & $-2,89 \%$ & 1,41 & 13 \\
Mediano & $7,38 \%$ & $-45,06 \%$ & $-11,91 \%$ & 0,67 & 15 \\
Alto & $4,63 \%$ & $-17,51 \%$ & $-19,89 \%$ & 0,92 & 7 \\
\hline Total & $6,96 \%$ & $-9,57 \%$ & $-10,16 \%$ & 0,99 & 35 \\
\hline
\end{tabular}

Considerando que la mayoría de las IPO's del estudio son empresas pequeñas y con razones $B E / M E$ mediano y bajos, se estableció que a diferencia de lo planteado por Fama (1998) y Brav (2000), el bajo desempeño de las IPO's no es un comportamiento típico para este tipo de empresas.

Es decir, los rendimientos anormales negativos de las IPO's son más importantes para las empresas pequeñas y con razones $B E / M E$ medios y altos, esto es, empresas pequeñas con negocios maduros con relativamente escasas expectativas de crecimiento.

\section{Conclusiones}

En lo que respecta al análisis de corto plazo, las IPO's tuvieron un underpricing en promedio igual a $6.77 \%$, que si bien es alto, resultó ser significativamente más bajo que los retornos iniciales que se han reportado en otros países.

Si bien entre los años 1993 y 2000, dos tercios de las acciones que se abrieron a Bolsa perdieron más de la mitad de su valor, los resultados indican que la existencia o no de retornos anormales a largo plazo depende de diversas cuestiones metodológicas. La literatura ofrece una considerable variedad en medidas de retornos anormales y de pruebas estadísticas que han sido utilizados para detectar la existencia de retornos anormales a largo plazo. Dado que ninguno de los métodos utilizados en diversos estudios resulta completamente confiable, en esta investigación se optó por utilizar tres metodologías diferentes a fin de dar mayor robustez a los resultados obtenidos.

Mediante el uso de HPR, se constató la existencia de retornos anormales negativos para ciertas carteras equiponderadas de IPO's. De esta forma, se estableció que las carteras equiponderadas de IPO's que el primer día emitieron montos pequeños, así como las carteras conformadas por IPO's que presentaron bajos retornos iniciales, mostraron cierta evidencia de un mal desempeño a largo plazo. Sin embargo, no se logró establecer ninguna asociación "lineal" entre estas dos anomalías. 
Por otro lado, al calcular los CAR se pudo establecer cierta evidencia de retornos anormales estadísticamente significativos para una "cartera temporal" equiponderada de IPO's. Estos últimos, siguieron un patrón sistemático a lo largo de la ventana de 36 meses cuando se utilizó como benchmark el IPSA, no así cuando se utilizó el IGPA. Se concluyó que el IPSA no era un grupo de control idóneo, por ser un índice selectivo que dista mucho de ser una inversión alternativa y comparable con una cartera de IPOs. En el periodo en estudio los IPO's se concentraron principalmente en empresas pequeñas y con razones $\mathrm{BE} / \mathrm{ME}$ bajos y medianos.

El modelo de CAPM de Merton, así como el de tres factores de Fama y French, reafirman la utilización del IGPA como grupo de control, al resultar ser el retorno en exceso de éste sobre la $r_{f}$, un factor relevante al explicar el retorno en exceso de una cartera equiponderada de IPO'S sobre la $r f$. El modelo de tres factores de Fama y French constató la inexistencia de retornos anormales negativos medios para una cartera equiponderada de toda las IPO's de la muestra en estudio. Sin embargo, un análisis desagregado según tamaño y razón $\mathrm{BE} / \mathrm{ME}$, mediante el uso de HPR's permitió obtener evidencia de un bajo desempeño a largo plazo para una cartera conformada por empresas pequeñas y con razones elevadas como medianos, es decir, empresas pequeñas con escasas expectativas de crecimiento.

\section{Bibliografía}

Aggarwal, R., R. Leal and L. Hernández (1991). The aftermarket performance of initial public offerings in Latin America. Financial Management, 22, pp. 42-53.

Barber, B. and J. Lyon (1997). Firm Size, Book-to-Market Ratio, and Security Returns: A Holdout Sample of Financial Firms. Journal of Finance, 52(2), pp. 875-883.

Brav, A. (2000). Inference in Long-Horizon Event Studies: a Bayesian Approach with Application to Initial Public Offerings. Journal of Finance, 55(5), pp. 1979-2016.

Beatty R. and J. Ritter (1986). Investment Banking, Reputation and the Underpricing of Initial Public Offerings. Journal of Financial Economics, 15, pp. 213-232.

Fama, E. (1998). Market efficiency, Long-Term Returns and Behavioral Finance. Journal of Financial Economics, 49, pp. 283-306.

Fama, E. and K. French (1992). The Cross Section of Expected Stock Returns. The Journal of Finance, $47(2)$, pp. 427-465.

Fama, E. and K. French (1993). Common Risk Factors in the Returns of Stocks and Bonds. Journal of Financial Economics, 33, pp. 3-56.

Grinblatt, M. and C. Y. Hwang (1989). Signalling and the Pricing of New Issues. Journal of Finance, 44(29), pp. 393-420.

Ibbotson, R. G. (1975). Price Performance of Common Stock Now Issues. Journal of Financial Economics, 2, pp. 235-272.

Ibbotson, R. G. and J. F. Jaffe (1975). Hot Issue Markets. Journal of Finance, 30(4), pp. 1027-1042.

Ibbotson R. G., J. L. Sindelar and J. Ritter (1988). Initial Public Offerings. Journal of Applied Corporate Finance, 1(2), pp. 37-45.

Loughran, T. and J. Ritter (2000). Uniformly Least Powerful Test of Market Efficiency. Journal of Financial Economics, 55, pp. 361-389.

Loughran, T., J. Ritter, and K. Rydqvist (2001). Initial Public Offerings: International Insights. Pacific Basin Finance Journal, 2, pp. 165-199.

Loughran, T. and J. Ritter (1995). The New Issues Puzzle. Journal of Finance, 50(1), pp. 23-51. 
Lyon, J., B. Barber, and C. Tsai (1999). Improved Methods for Tests of Long-Run Abnormal Stock Returns. Journal of Finance, 54(1), pp. 165-201.

Ritter, J. and I. Welch (2002). A Review of IPO activity, Pricing, and Allocations. Journal of Finance, 57(4), pp. 1795-1828.

Ritter, J. (1984). The Hot Issue Market of 1980. Journal of Business, 57(2), pp. 215-240.

Ritter, J. (1991). The Long Run Desempeño of IPO's. Journal of Finance, 42, pp. 365-394.

Ritter, J. (1998). Initial Public Offerings. Contemporary Finance Digest, 2 (1), pp. 5-30.

Rock, K. (1986). Why New Issues Are Underpriced. Journal of Financial Economics, 15, $187-212$.

Tinic, S. (1988). Anatomy of IPO's of Common Stock. Journal of Finance, 43(4), pp. $789-822$.

Welch, I. (1989). Seasoned Offerings, Imitation Costs, and the Underpricing of Initial Public Offerings. Journal of Finance, 44(2), pp. 421-450.

Womack, K. L. (1996). Do Brokerage Analysts' Recommendations Have Investment Value?. Journal of Finance, 51(1), pp. 137-167. 